ESAIM: M2AN 47 (2013) 935-960

DOI: $10.1051 / \mathrm{m} 2 \mathrm{an} / 2012052$
ESAIM: Mathematical Modelling and Numerical Analysis

www.esaim-m2an.org

\title{
MODELING OF THE OXYGEN TRANSFER IN THE RESPIRATORY PROCESS *
}

\author{
SÉBastien Martin ${ }^{1,2}$ And Bertrand Maury ${ }^{2}$
}

\begin{abstract}
In this article, we propose an integrated model for oxygen transfer into the blood, coupled with a lumped mechanical model for the ventilation process. Objectives. We aim at investigating oxygen transfer into the blood at rest or exercise. The first task consists in describing nonlinear effects of the oxygen transfer under normal conditions. We also include the possible diffusion limitation in oxygen transfer observed in extreme regimes involving parameters such as alveolar and venous blood oxygen partial pressures, capillary volume, diffusing capacity of the membrane, oxygen binding by hemoglobin and transit time of the red blood cells in the capillaries. The second task consists in discussing the oxygen concentration heterogeneity along the path length in the acinus. Method. A lumped mechanical model is considered: a double-balloon model is built upon physiological properties such as resistance of the branches connecting alveoli to the outside air, and elastic properties of the surrounding medium. Then, we focus on oxygen transfer: while the classical [F.J. Roughton and R.E. Forster, J. Appl. Physiol. 11 (1957) 290-302]. approach accounts for the reaction rate with hemoglobin by means of an extra resistance between alveolar air and blood, we propose an alternate description. Under normal conditions, the Hill's saturation curve simply quantifies the net oxygen transfer during the time that venous blood stays in the close neighborhood of alveoli (transit time). Under degraded and/or exercise conditions (impaired alveolar-capillary membrane, reduced transit time, high altitude) diffusion limitation of oxygen transfer is accounted for by means of the nonlinear equation representing the evolution of oxygen partial pressure in the plasma during the transit time. Finally, a one-dimensional model is proposed to investigate the effects of longitudinal heterogeneity of oxygen concentration in the respiratory tract during the ventilation cycle, including previous considerations on oxygen transfer. Results. This integrated approach allows us to recover the right orders of magnitudes in terms of oxygen transfer, at rest or exercise, by using well-documented data, without any parameter tuning or curve fitting procedure. The diffusing capacity of the alveolar-capillary membrane does not affect the oxygen transfer rate in the normal regime but, as it decreases (e.g. because of emphysema) below a critical value, it becomes a significant parameter. The one-dimensional model allows to investigate the screening phenomenon, i.e. the possibility that oxygen transfer might be significantly affected by the fact that the exchange area in the peripheral acinus poorly participates to oxygen transfer at rest, thereby providing a natural reserve of transfer capacity for exercise condition. We do not recover this effect: in particular we show that, at rest, although the oxygen concentration is slightly smaller in terminal alveoli, transfer mainly occurs in the acinar periphery.
\end{abstract}

Mathematics Subject Classification. 35Q92, 76Z05, 76R50, 92C35, 92C50.

Received December 21, 2011.

Published online June 13, 2013.

Keywords and phrases. Oxygen transfer, ventilation, lung diffusion capacity, advection-diffusion equation.

* This work was partially funded by the ANR projects: M3RS ANR-08-JCJC-0013-01 and OxHelease ANR-11-TECS-006.

1 INRIA Paris Rocquencourt, REO project - BP 105, 78153 Le Chesnay cedex, France

2 Laboratoire de Mathématiques d'Orsay, Université Paris-Sud, 91405 Orsay cedex, France. bertrand.maury@math.u-psud.fr 


\section{INTRODUCTION}

The respiratory system is designed to achieve two main functions: oxygen transfer from the outside air to the blood, and expulsion of carbon dioxide from the blood to the air. Those transfers are achieved through passive diffusion across a membrane which separates both phases (the gaseous air and the liquid blood), at an instantaneous rate which depends on the difference in partial pressures, the area of the exchange surface, and its properties in terms of diffusion. As this diffusion tends to reduce the partial pressure difference, a constant renewal must be made on both sides of the membrane. Renewal of air is achieved by the ventilation process, which consists in periodic inspiration-expiration cycles that provide the inside of the lung with fresh air, whereas venous blood is periodically pumped onto the exchange zone by the heart.

The exchange area is the boundary of a huge collection of small cavities (around 300 million units), called alveoli, which scale around a quarter millimeter, which makes an exchange area of about $100 \mathrm{~m}^{2}$, see $[43,47]$ for typical values. Each of this alveolus is surrounded by a network of very small blood vessels, called capillaries, whose diameter is about 5 to $10 \mu \mathrm{m}$, see [47]. Gas exchanges occur through the alveolar-capillary membrane, which is around $0.5 \mu \mathrm{m}$. The alveoli are connected to the outside world through the respiratory tract, which is an assembling of interconnected pipes following a dyadic-tree structure that can be described as follows [43, 47]: the trachea (with a diameter around $2 \mathrm{~cm}$ ) divides onto two sub-branches, which divide further onto smaller branches, and so on, up to 23 levels of bifurcations, although the total number of generations depends on the subject [45]. The first generations are purely conductive and exchanges do not take place before generation 16 or 17: bronchi are lined up with alveoli from generation 17 to the end but alveoli are quite dispersed in generation 17 and their number increases with the generation, see $[18,31,39,45]$. Beyond that point, all branches are lined up with alveoli, up to the last generation. Figure 1 gives an overview of the different zones of the respiratory system, from the trachea to alveolar-capillary membrane, together with orders of magnitude in terms of dimensions. For a normal person, under resting conditions, the volume of air available for gas exchange corresponds to the alveolar zone, it sums up around 2.5 to $3 \mathrm{~L}$. This volume is easily accessible to measurement. The volume of blood in the neighborhood of the exchange surface is called the capillary volume, it is estimated around $70 \mathrm{~mL}$, see $[4,18,47]$, although it is much less accessible to direct measurement and usually estimated by mean of indirect methods which rely on some modeling assumptions. The efficiency of the lung as oxygen exchanger is well documented in the literature: the standard flow of oxygen that goes into the bloodstream of an adult ranges between $250 \mathrm{~mL} \mathrm{~min}{ }^{-1}$ and $400 \mathrm{~mL} \mathrm{~min}^{-1}$ under resting conditions, and may reach $3 \mathrm{~L} \mathrm{~min}^{-1}$ during exercise, and even more for well-trained athletes, $[18,31]$, all the values above expressing the quantity of oxygen as the volume it would occupy as a gas at standard temperature and pressure.

As far as one considers healthy lungs, the apparent geometric complexity of the respiratory tract is not a crucial issue in terms of oxygen transfer: the dyadic structure makes it relevant to consider that, under normal conditions, all quantities shall be approximately constant in each generation, so that the overall phenomenon is in some way one-dimensional: the only relevant dimension is the longitudinal one, i.e. the path length expressing the distance from the entrance of the system, nose or entrance of the trachea.

Far more crucial are the two following issues, on which the present paper focuses:

1. The chemistry of the oxygen-hemoglobin reaction is complex; in particular the relation between oxygen partial pressure in the plasma (which controls the rate at which oxygen crosses the membrane) and the actual content of oxygen in the blood (oxygen is mainly present as combined with hemoglobin) is nonlinear.

2. The concentration of oxygen is not uniform overall the lungs. Oxygen is advected by the global flow of air induced by ventilation, and it also diffuses in the air. Advection is predominant in the upper part of the tree, whereas transport is essentially diffusive in the acinar region. As oxygen diffuses away through the membrane, concentration gradients appear. Diffusion tends to uniformize the concentration, but as we shall see the diffusion characteristic times are of the order of magnitude of the ventilation cycle duration, during which air is renewed. As a consequence, significant differences in oxygen concentration are likely to appear, and influence the overall transfer process. 

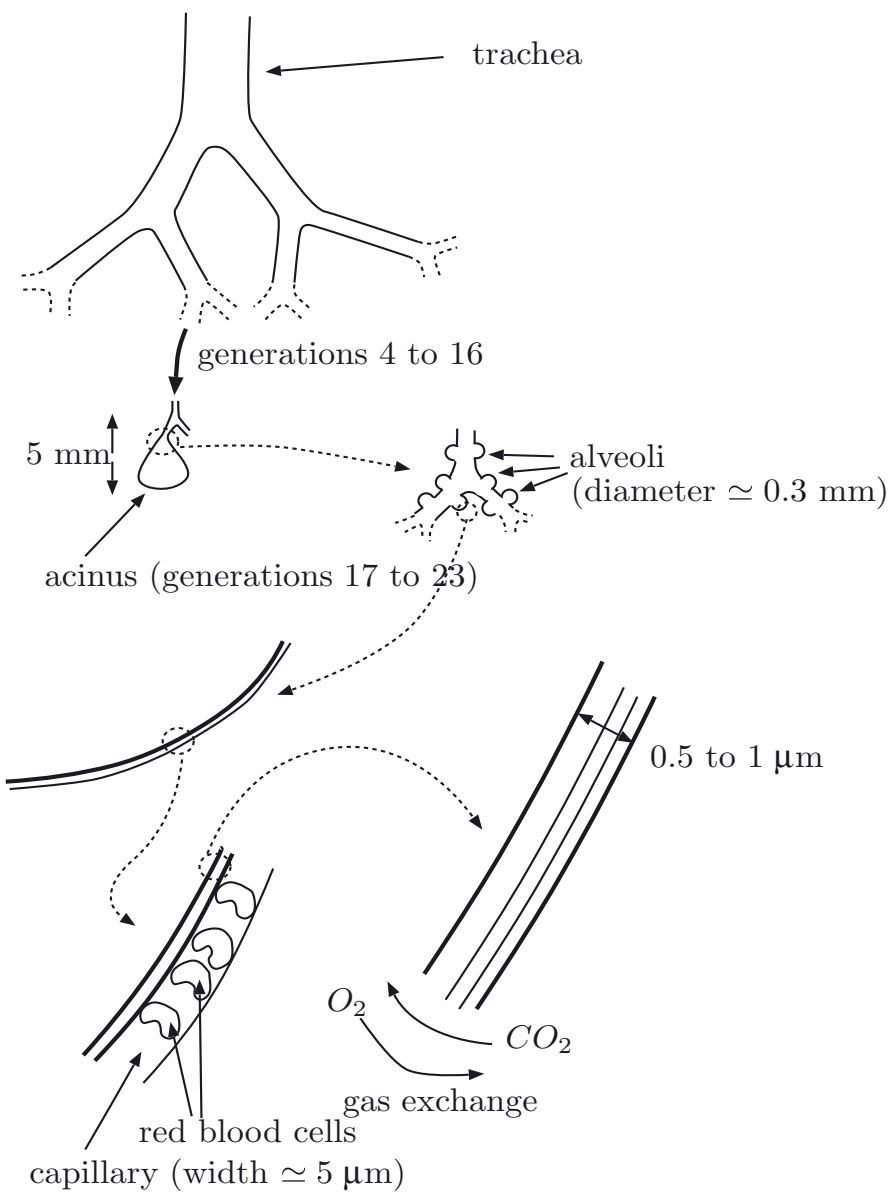

FiguRE 1. Overall view of the process of oxygen transfer into blood: from the bronchial tree to the capillaries.

Point (1) is addressed as follows. At a macroscopic level, the oxygen transfer into the blood is commonly quantified by the pulmonary diffusing capacity for oxygen. This quantity $D_{L}$ is defined (see [8,43-45], and the pioneering work by Roughton and Forster [33] on carbon monoxide) by the following relation

$$
\dot{V}_{O_{2}}=D_{L}\left(P_{\mathrm{A}}-\bar{P}_{c}\right) \text {, }
$$

where $P_{\mathrm{A}}$ is the partial pressure of oxygen in the alveolar air, $\bar{P}_{c}$ is the mean partial pressure of oxygen in the capillary, and $\dot{V}_{\mathrm{O}_{2}}$ the oxygen transfer rate (usually expressed in $\mathrm{mL} \mathrm{min}^{-1}$ ). Note that some authors (see e.g. [8]) tend to favor the term transfer factor for $D_{L}$. The associated resistance is usually decomposed onto two contributions (see e.g. $[33,45])$ :

$$
\frac{1}{D_{L}}=\frac{1}{D_{\mathrm{m}}}+\frac{1}{\theta V_{c}}
$$

where $D_{\mathrm{m}}$ stands for the alveolar-capillary membrane diffusing capacity also called diffusive conductance in [7], $V_{c}$ is the capillary volume, and $\theta$ accounts for the binding rate of hemoglobin with oxygen. The physiological meaning of this latter quantity is not fully understood, and its actual measurement is delicate, see [43]. Besides, actual measurements of $D_{L}$ may lead to values which depend on $P_{\mathrm{A}}$ itself, see e.g. [2], although its definition relies on equation (1), which rules out such a dependance. Our motivation to propose a model which does 
not rely on this parameter $\theta$ is based on the following arguments. As we will detail in Section 2, kinetics of oxygen-hemoglobin interaction is such that partial pressures will reach equilibrium before the available time is attained, under normal conditions. Indeed, the time that blood remains in the neighborhood of the exchange zone is about three times larger than the time it takes to achieve balance. As a consequence, a decrease of $D_{\mathrm{m}}$ may well decelerate the process, it will not be followed by any observable consequence on the oxygen transfer rate, whereas it decreases $D_{L}$ according to equation (2). The striking consequence of this simple remark is that the permeability of the membrane (which is related to the membrane diffusing capacity $D_{\mathrm{m}}$ ) does not appear as a relevant parameter to model oxygen transfer under normal conditions. In other words, it is not an observable parameter in the standard regime. Of course a strong decrease of $D_{\mathrm{m}}$ will eventually be harmful to the overall process, and we shall quantify this phenomenon of diffusion limitation, but consequences will not be observed if the changes are small. Our second remark pertains to the pulmonary diffusing capacity (or transfer factor) $D_{L}$, which states a linear relation between oxygen transfer rate and difference of pressures. As it has been extensively discussed in the literature, the oxygen-hemoglobin reaction can be described by the so-called Hill's saturation curve, which relates the partial pressure of oxygen in the plasma to the actual quantity of oxygen carried by hemoglobin. The Hill's curve is far from being linear in the zone of interest. In particular, as we shall see, it is almost flat in the neighborhood of alveolar partial pressure of oxygen, whereas it is stiff for smaller partial pressures, like in the venous blood. As another striking fact, the oxygen transfer rate is quasi independent from oxygen partial pressure in the alveoli, $P_{\mathrm{A}}$, and the dependency is far from being linear, because of the sigmoid pattern of the saturation curve. In the Roughton and Forster approach [33] described by equation (2), parameter $\theta$ carries information on all those non-linear phenomena (mainly hemoglobin saturation and diffusion kinetics), in a very indirect manner. The approach we propose does not rely on a representation of chemical reactions as an extra resistance in series with the membrane, but rather incorporates the chemical reaction with hemoglobin by means of an expression of the net transfer based on the Hill's curve expressing the saturation function, together with an evolution equation on plasma oxygen partial pressure, to account for possible diffusion limitation.

Point (2) has been addressed more recently as diffusion screening phenomenon, as results tend to provide a new evidence of the existence of diffusional screening at the acinar level, implying permanent spatial inhomogeneity of oxygen and carbon dioxide partial pressure. This phenomenon has been primarily studied in abstract geometric models of the acinus such as the Hilbert acinus [35] then extended to 3D model geometries such as the Kitaoka acinus [12-14] and further discussed in [34]. In the meanwhile, [20] suggests that the lung operates in the partial-screening regime, close to the transition to no screening, for respiration at rest and in the no-screening regime for respiration at exercise. The approach we propose here provides new insight on those issues. We shall disregard here the Péclet number approach proposed in the mentioned references, and use a one-dimensional model of the oxygen distribution in the lung to investigate the relative weights of advection and diffusion in the quantity of oxygen which is actually transferred to the blood.

\section{MechanicAl MODEL}

Although the mechanical behavior of the lung is not our prior objective here, we aim at investigating the influence of some mechanical parameters upon the oxygen transfer, which makes it necessary to propose a global system controlled by the actual action performed by a patient, namely the contraction of the diaphragm and, possibly, the abdominal muscles. Mechanical modeling of the ventilation process involves two main ingredients: resistance of the branches connecting alveoli to the outside air, and elastic properties of the surrounding medium. We present here a double-balloon model, see Figure 2, which will make it possible to distinguish between the forcing term resulting from the contraction of the diaphragm and the pleural pressure, which is directly accessible to measurement and commonly considered to be close to the esophageal pressure, which is actually measured in practice [40]. The inner balloon in the model accounts for the lung itself, which is considered as a nonlinear elastic medium which tends to reduce its volume $V$ (actually the volume of air that it contains) close to 0 , because of elastic properties of the fibers which constitute the parenchyma, together with surface tension forces 


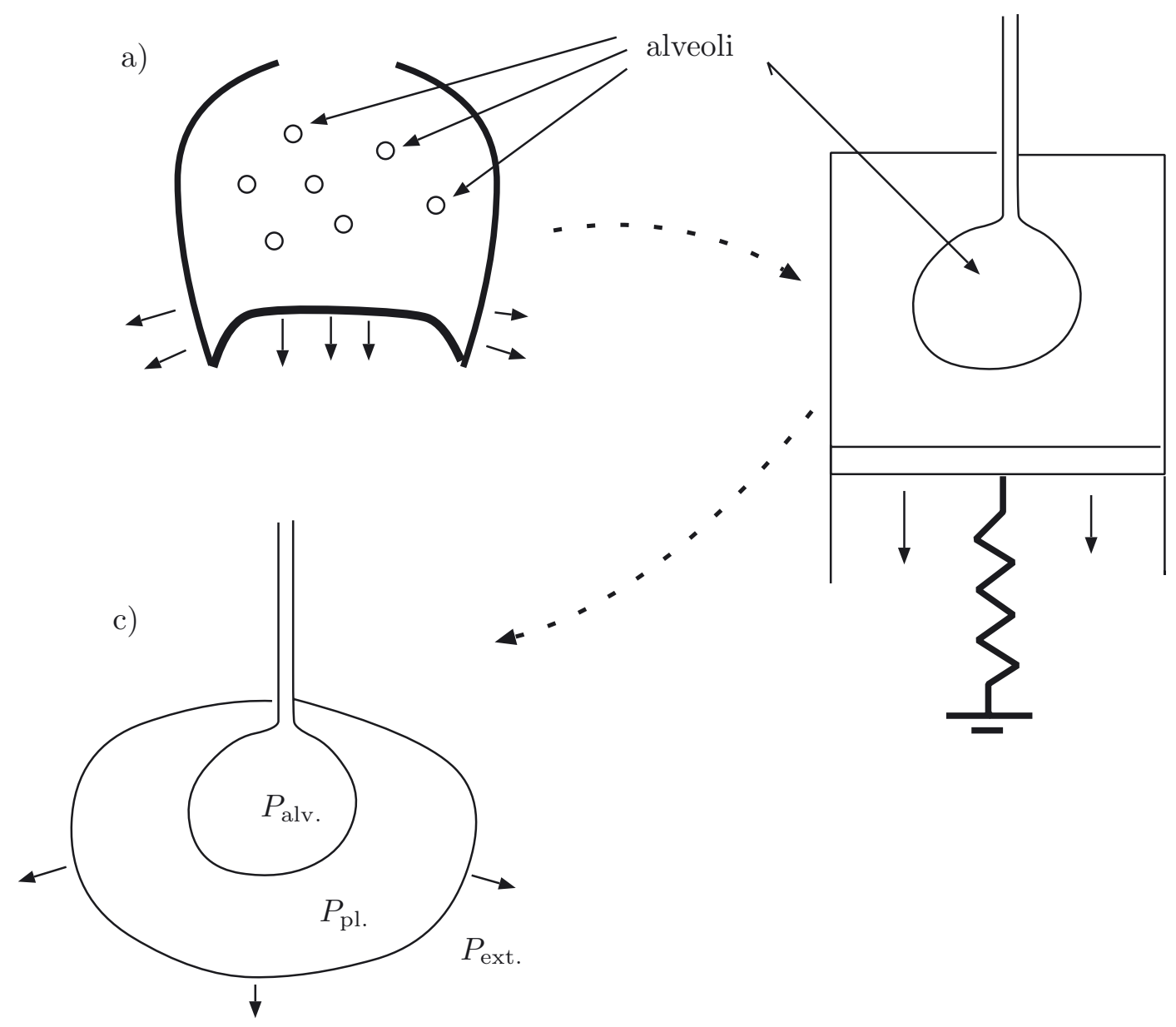

b)

FiguRE 2. Double-balloon model.

which tend to reduce the size of alveoli. The elastic behavior of this inner balloon will be represented by a function $\varphi_{L}$ which relates the volume to the difference of partial pressure in the alveoli and in the pleura:

$$
P_{\text {alv. }}-P_{\text {pl. }}=\varphi_{L}(V),
$$

where $P_{\text {alv }}$. is the pressure of air in the alveoli, and $P_{\mathrm{pl}}$. the pleural pressure.

The outer balloon corresponds to the thoracic cage, which would spontaneously tend to be slightly larger than it is at rest, as it is maintained smaller by the negative pleural pressure. Its behavior is described as previously by a function $\varphi_{C}$ (note that the pleural pressure is now inside the balloon, whereas it was outside the inner balloon). As the flesh within the lung is essentially incompressible (volume variations correspond to variations of the gaseous and deformable parts, i.e. essentially the alveoli), the mechanical behavior can be expressed in terms of the volume of air in the lung, as previously:

$$
P_{\text {pl. }}-P_{\text {ext. }}=\varphi_{C}(V),
$$

where $P_{\text {ext. }}$ is the pressure "outside" the cage, often referred as airway opening pressure or transpulmonary pressure, see e.g. $[4,36]$. Note that this quantity is not accessible to direct measurement. It accounts for the effort of the diaphragm $\left(P_{\text {ext. }}<0\right.$ for inspiration), and possibly muscles of the abdomen during forced expiration $\left(P_{\text {ext. }}=0\right.$ for passive expiration and $P_{\text {ext. }}>0$ for forced expiration $)$. 


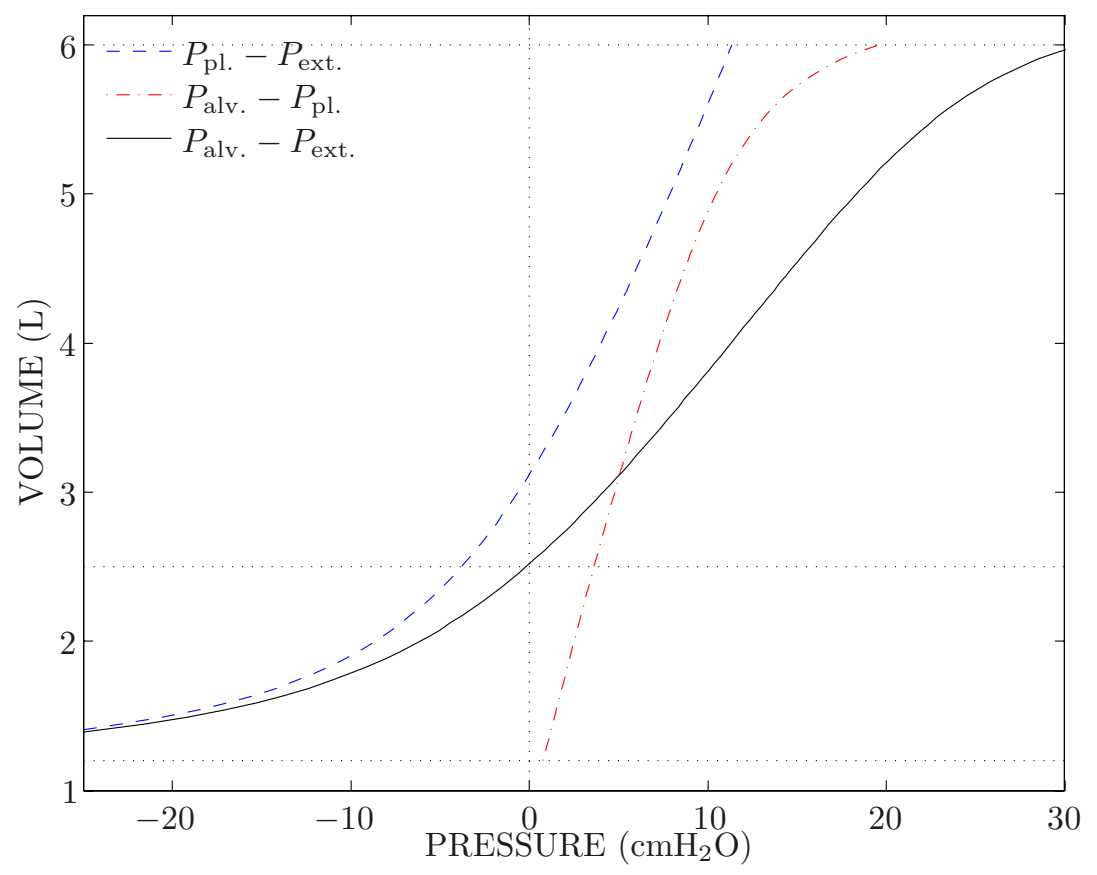

FiguRE 3. Elastic behavior of the lung: relation between the lung volume $V$ and the difference of pressure $\Delta P$ (from [1]) for typical values of $V_{\mathrm{RV}}=1.2 \mathrm{~L}, V_{\mathrm{FRC}}=2.5 \mathrm{~L}$ and $V_{\mathrm{TLC}}=6 \mathrm{~L}$.

A simple ODE model can be obtained by eliminating the pleural pressure between both equations, this double-balloon approach providing the possibility to recover the pleural pressure as an outcome of the model. Furthermore, this approach allows to account for changes in the elastic properties of the lung and of the thoracic cage independently. By simply adding both equations, one obtains

$$
P_{\text {alv. }}-P_{\text {ext. }}=\varphi_{L}(V)+\varphi_{C}(V):=\varphi(V) .
$$

We now write the generalized Poiseuille law for the whole tree, which relates the flux $\dot{V}$ to the difference of pressure between alveoli and the outside world (considered at pressure 0 ):

$$
0-P_{\text {alv }}=R \dot{V} .
$$

We finally obtain

$$
R \dot{V}+\varphi(V)=-P_{\text {ext. }},
$$

The assumption we shall make on $\varphi$ are based in Figure 3, which represents the reciprocal (in the pressurevolume plane) functions of $\varphi_{L}$ (dashed curve on the right), $\varphi_{C}$ (dashed curve on the left), and $\varphi$ (central bold curve). On each of those curves, the slope corresponds to the local compliance, and its reciprocal to the elastance.

Before giving an explicit expression for $\varphi$, let us first note that, as function $\varphi$ is increasing (which simply express that each balloon of the model tends to get larger as the inner pressure increases), it blows up to $+\infty$ (resp. $-\infty$ ) as the volume goes to some maximal value $V_{\mathrm{TLC}}$ corresponding to the Total Lung Capacity (resp. some minimal value $V_{\mathrm{RV}}$ corresponding to the Residual Volume). Function $\varphi$ vanishes at a unique volume which corresponds to static equilibrium corresponding to the end of a passive expiration. According to physiological tradition, we shall denote this value by $V_{\mathrm{FRC}}$ corresponding to the Functional Residual Capacity. Note that the linear model which is commonly used in practice, see e.g. [4], can be recovered in a natural way by writing, in 
the neighborhood of $V_{\mathrm{FRC}}$,

$$
\varphi(V) \simeq \underbrace{\varphi\left(V_{\mathrm{FRC}}\right)}_{=0}+\varphi^{\prime}\left(V_{\mathrm{FRC}}\right)\left(V-V_{\mathrm{FRC}}\right)
$$

which leads to

$$
R \dot{V}+E V=E V_{\mathrm{FRC}}-P_{\text {ext. }},
$$

where $E=\varphi^{\prime}\left(V_{\mathrm{FRC}}\right)$ is the elastance at $V_{\mathrm{FRC}}$, and $-E V_{\mathrm{FRC}}$ corresponds to the negative pleural pressure at rest which balances elastic forces which tend to reduce the volume of air in the lung. To obtain a non-linear model which is compatible with the experimental data near $V_{\mathrm{FRC}}$, and reproduces the high stiffness of the system near its extremal values $V_{\mathrm{RV}}$ and $V_{\mathrm{TLC}}$, we propose the following construction: $\varphi$ is built as a sum of two hyperbolic functions, which are singular at $V_{\mathrm{RV}}$ and $V_{\mathrm{TLC}}$, respectively, then translated to recover the right value for $V_{\mathrm{FRC}}$ which is commonly measured in medical practice, and then multiplied by a factor to obtain the right value for the elastance near $V_{\mathrm{FRC}}$. More precisely, considering a patient for which $V_{\mathrm{RV}}, V_{\mathrm{TLC}}, V_{\mathrm{FRC}}$ and elastance $E$ near $V_{\mathrm{FRC}}$ are known, we define $\varphi$ as

$$
\varphi(V)=\lambda\left(\frac{1}{V_{\mathrm{TLC}}-V}-\frac{1}{V-V_{\mathrm{RV}}}-\left(\frac{1}{V_{\mathrm{TLC}}-V_{\mathrm{FRC}}}-\frac{1}{V_{\mathrm{FRC}}-V_{\mathrm{RV}}}\right)\right),
$$

with

$$
\lambda=E\left(\frac{1}{\left(V_{\mathrm{TLC}}-V_{\mathrm{FRC}}\right)^{2}}+\frac{1}{\left(V_{\mathrm{FRC}}-V_{\mathrm{RV}}\right)^{2}}\right)^{-1} .
$$

Note that this approach makes it possible to recover the pleural pressure $P_{\mathrm{pl}}$. as

$$
P_{\text {pl. }}(t)=P_{\text {ext. }}(t)+\varphi_{C}(V(t))
$$

Remark 1.1. Let us note that, in practice, $V_{\mathrm{TLC}}$ and $V_{\mathrm{RV}}$ are measured for finite effort so that blow up is actually considered for a maximal volume which is slightly larger than the actual $V_{\text {TLC }}$, and a minimal volume which is smaller than $V_{\mathrm{RV}}$. In our model, we assume that $V_{\mathrm{TLC}}$ and $V_{\mathrm{RV}}$ actually correspond to an infinite respiratory system pressure, for the sake of simplicity. This modification does not significantly affect the aspect of the elastic response for the regimes we considered (rest or exercise) but should be taken into account in a more accurate way in the case of forced maneuvers, in the context of spirometry for instance.

\section{LUMPED OXYGEN TRANSFER MODEL}

In [4], Ben-Tal presents a hierarchy of lumped models of the human lungs for both the mechanical behavior of the lung, considered as a single compartment container, and the description of gas exchange. In particular, a large overview of the literature on the different phenomena involved in the gas exchange process is described. In a more general context, $[18,21,32,47]$ and references therein describe at various levels of details the main principles of pulmonary ventilation, pulmonary circulation, then physical principles of gas exchange such as diffusion of oxygen and carbon dioxide through the respiratory membrane: the basic principles leading to the oxygen uptake along the pulmonary capillary that are presented implicitly deal with an average lumped model, although highlighting the evidence of spatial heterogeneity of the gas transfer.

\subsection{The reaction-diffusion model}

The simplest model for oxygen uptake in the blood is obtained as follows: the empty space in the lung is considered a single balloon with volume $V$, with uniform oxygen concentration $t \mapsto c(t)$. The latter is expressed as a dimensionless number in $[0,1]$, so that the volume of oxygen in the balloon is $c V$. During inspiration, the rate of oxygen which enters the balloon is $c^{0} \dot{V}>0$, where $c^{0}=0.2$ corresponds to fresh air. During expiration, 
it becomes $c \dot{V}<0$ as oxygen is rejected. Now denoting by $Q$ the flux of oxygen which diffuses onto the blood, the evolution of the volume of oxygen in the balloon can be expressed

$$
\frac{\mathrm{d}}{\mathrm{d} t}(c V)=H(\dot{V}) c^{0} \dot{V}+(1-H(\dot{V})) c \dot{V}-Q,
$$

where $H(\cdot)$ is the Heaviside graph (characteristic function of $\mathbb{R}^{+}$). One obtains

$$
\dot{c}=\frac{1}{V}\left(\dot{V}\left(c^{0}-c\right) H(\dot{V})-Q\right) .
$$

To estimate $Q$, we introduce the transit time $\tau_{b}$ as the time that a red blood cell spends in the exchange zone, and we consider, as in [38], that the following phenomenon happens every $\tau_{b}$ : a quantity $V_{c}$ of venous blood is brought instantaneously in the neighborhood of alveoli. It stays there during $\tau_{b}$, allowing exchanges to take place, then it is evacuated and replaced by the same amount of venous blood. The net oxygen transfer is the total quantity after the cycle minus its initial value. We furthermore consider Bohr assumptions, see [5], considering that the alveolar oxygen partial pressure $P_{\mathrm{A}}$ is constant and uniform, the diffusing properties of the blood-gas barrier are constant and uniform along the capillaries, and the chemical reaction of oxygen with hemoglobin is instantaneous. According to this set of assumptions, the concentration $\gamma$ of oxygen in the plasma is considered to be uniform at any time over the whole capillary volume $V_{c}$. The concentration of oxygen in blood, denoted $\gamma$ can be expressed in terms of partial pressure, by means of solubility of oxygen in the plasma, denoted $\sigma$ :

$$
\gamma:=\sigma P
$$

with $\sigma=1.4 \times 10^{-6} \mathrm{~mol} \mathrm{~L}^{-1} \mathrm{mmHg}^{-1}$. The total partial concentration of oxygen contained in the considered volume of blood is $\sigma P$ to which one must add a highly significant contribution due to the fact that some oxygen has been captured by hemoglobin. This latter contribution writes $4 C f(P)$, where $C$ is the total concentration of hemoglobin (in both native and combined forms), $f(P) \in[0,1]$ quantifies the saturation of hemoglobin, and 4 is the maximal number of oxygen molecules that a molecule of hemoglobin may carry. According to various sources, see e.g. [22], this saturation function corresponds to Hill's curve, and can be expressed as

$$
f(P)=\frac{P^{n}}{\tilde{P}^{n}+P^{n}},
$$

with $n=2.5$ and $\tilde{P} \simeq 26 \mathrm{mmHg}$ [22]. The fact that $n$ is larger than 1 can be explained by the cooperative character of the complex reaction between hemoglobin and oxygen, see e.g. [11], i.e. the fact that, once a first molecule of oxygen has been captured by hemoglobin, others bind more easily. This explains the sigmoid character of this curve, but the actual value has to be obtained experimentally by a curve fitting procedure, and it varies in the literature between $n=2.5$ and $n=2.8$ (it is 2.5 in [22], and 2.7 in [5]). Some authors, e.g. $[38,48]$, favor an alternative expression, namely the Kelman dissociation curve [23] which is built as the quotient between two fourth order polynomials, whose coefficients are determined by curve fitting according to experimental data.

Note that $f$ is very small and flat for small values of $P$, and saturates toward 1 for large values. The stiff transition between extreme situations takes place around $\tilde{P}=26 \mathrm{mmHg}$. As the partial pressure of oxygen in the venous blood $P_{\mathrm{V}}$ is about $40 \mathrm{mmHg}$, possibly $30 \mathrm{mmHg}$ during exercise, all phenomena take place on the right-hand side of this transition value, in a zone where the function is concave. Figure 4, which corresponds to the Hill's curve, see equation (4), plots the saturation function $P \mapsto f(P)$. At rest (resp. exercise) in a healthy situation, venous blood enters the exchange zone with hemoglobin saturated at $75 \%$ (resp. 60\%) and goes out from the capillaries with hemoglobin saturated at $97 \%$.

The instantaneous flux of oxygen through the membrane by passive diffusion is proportional to the difference of partial pressures $\left(P_{\mathrm{A}}\right.$ in the alveoli, and $P$ in the blood), and the relation involves a crucial quantity $D_{\mathrm{m}}$ already mentioned in the introduction as alveolar-capillary membrane diffusing capacity. The concentration of 


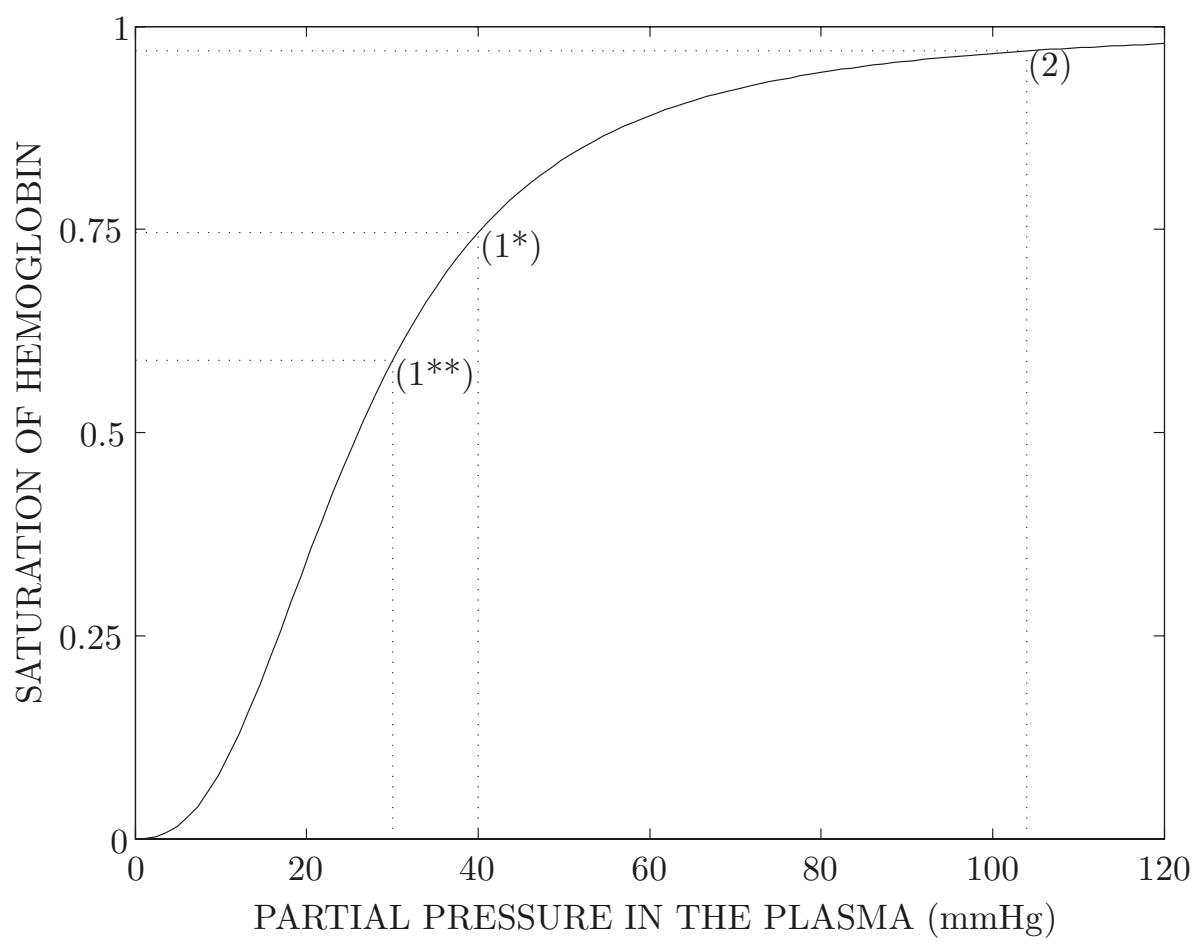

FIGURE 4. Oxygen-hemoglobin dissociation curve: partial pressure of oxygen in the bloodstream is about $40 \mathrm{mmHg}$ for incoming blood at rest $\left(1^{*}\right)$ and $30 \mathrm{mmHg}$ for incoming blood at exercise $\left(1^{* *}\right)$; it reaches $100 \mathrm{mmHg}$ in outcoming blood (2).

oxygen in the blood in both forms (free and captured by hemoglobin) writes $\sigma P+4 C f(P)$. If we denote by $V_{c}$ the capillary volume (i.e. volume of blood involved in the process that is under consideration), the balance on total quantity of oxygen writes

$$
V_{c} \frac{\mathrm{d}}{\mathrm{d} t}(\sigma P+4 C f(P))=D_{\mathrm{m}}\left(P_{\mathrm{A}}-P\right),
$$

which yields

$$
\left(1+4 C \sigma^{-1} f^{\prime}(P)\right) \dot{P}=\kappa\left(P_{\mathrm{A}}-P\right),
$$

with $\kappa:=D_{\mathrm{m}} /\left(\sigma V_{c}\right)$. If there were no hemoglobin, one would get an exponential relaxation of $P$ to $P_{\mathrm{A}}$, with a characteristic time $\kappa^{-1}$. But $f^{\prime}$ has a Gaussian shape around $\tilde{P}$, and the initial condition, corresponding to the concentration of oxygen in the venous blood, is $P_{\text {init. }}>\tilde{P}$. Therefore this term tends to decrease $\dot{P}$ at the beginning, then it goes down to 0 as $P$ increases to $P_{\mathrm{A}}$, so that this nonlinear terms tends to stiffen the evolution from $P_{\text {init. }}$ to $P_{\mathrm{A}}$, compared to a standard exponential relaxation.

Under standard conditions, the partial pressure of oxygen in venous blood is commonly considered to be $P_{\mathrm{V}}=40 \mathrm{mmHg}$, whereas $P_{\mathrm{A}}$ (considered constant) is around $100 \mathrm{mmHg}$. As for the membrane diffusing capacity $D_{\mathrm{m}}$, we consider the value proposed in $[4,7]$ :

$$
D_{\mathrm{m}} \simeq 21 \mathrm{~mL} \mathrm{~min}^{-1} \mathrm{mmHg}^{-1}=1.59 \times 10^{-5} \mathrm{~mol} \mathrm{~s}^{-1} \mathrm{mmHg}^{-1} .
$$

The capillary volume $[4,7]$ is $V_{c} \simeq 70 \mathrm{~mL}$. The concentration of hemoglobin is

$$
C \simeq 150 \mathrm{~g} \mathrm{~L}^{-1}=\frac{150 \mathrm{~g} \mathrm{~L}^{-1}}{68000 \mathrm{~g} \mathrm{~mol}^{-1}}=2.2 \times 10^{-3} \mathrm{~mol} \mathrm{~L}^{-1} .
$$




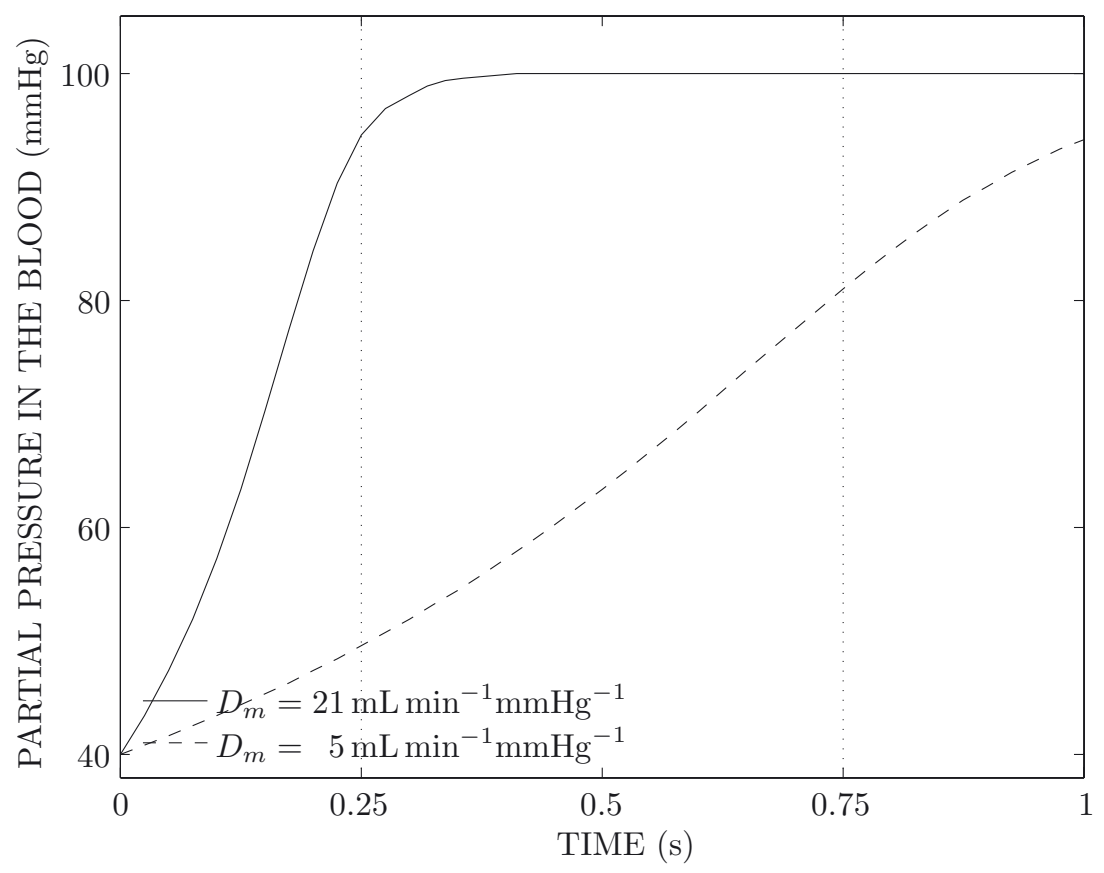

Figure 5. Evolution in time of partial pressure of oxygen $P$ in the capillaries; it reaches the value $P_{\mathrm{A}}^{*}$ at $t=0.75 \mathrm{~s}$ (at rest) or $t=0.25 \mathrm{~s}$ (at exercise).

Figure 5 represents the solution to equation (5), that was obtained numerically, expressed in terms of partial pressure. This explains why the partial pressure of oxygen in the blood during the $0.25 \mathrm{~s}$ that the process takes is usually represented as linear. Note that the presence of hemoglobin capturing oxygen slows down the increase of $P$ in the plasma. Indeed, the dimensionless quantity $1+4 C \sigma^{-1} f^{\prime}(\gamma)$ is much bigger than 1 in the neighborhood of $P \simeq 40 \mathrm{mmHg}$. Without hemoglobin, $P$ would balance with $P_{\mathrm{A}}$ almost instantaneously. But, as already mentioned, it drastically increases the quantity of oxygen in the blood when the balance is reached. Note that West [47] and Hughes [21] explicitly address in a similar way diffusion limitation (failure to achieve complete alveolar-end capillary equilibration) by highlighting comparison between rest regime and exercise regime.

Now let us focus on two aspects of the gas exchange: determination of the oxygen transfer rate, and diffusion limitation of oxygen transfer.

Oxygen transfer rate. Let us first notice that, assuming that the balance between partial pressures is attained during the time $\tau_{b}$ that venous blood spends in contact with alveoli, the quantity of oxygen which has been transferred does not depend on kinetics (it will be considered below). If we denote by $V_{c}$ the capillary volume expressing the total volume of blood in the neighborhood of the alveoli, the oxygen transfer rate can be derived under the assumption that we already mentioned: a quantity $V_{c}$ of venous blood is brought instantaneously in the neighborhood of the alveoli, then stays there during $\tau_{b}$, allowing exchanges to take place, and is finally evacuated and replaced by the same amount of venous blood; this phenomenon is periodically reproduced each time period $\tau_{b}$. In this context, the net oxygen transfer is the total quantity after the cycle minus its initial value. As the initial partial pressure of oxygen in the plasma is $P_{\mathrm{V}}$, and the final partial pressure is $P_{\mathrm{A}}$ (after a time $\tau_{b}$ ), and as the total concentration of oxygen in both free and combined forms at a given concentration in the plasma $\sigma P$ is $\sigma P+4 C f(P)$, we obtain the following formula for the oxygen transfer rate:

$$
Q=\frac{V_{c}}{\tau_{b}}[\sigma P+4 C f(P)]_{P_{\mathrm{V}}}^{P_{\mathrm{A}}}:=\frac{V_{c}}{\tau_{b}}\left(\sigma P_{\mathrm{A}}-\sigma P_{\mathrm{V}}+4 C\left(f\left(P_{\mathrm{A}}\right)-f\left(P_{\mathrm{V}}\right)\right)\right) .
$$


Remark 2.1. As pointed out in [43], the amount of oxygen carried by the plasma may reach about $10 \%$ of the total quantity when pure oxygen is breathed. Yet, under standard conditions, it is negligible ${ }^{3}$, meaning that $\sigma P$ is small in front of $4 C f(P)$, and the simpler expression

$$
Q \simeq 4 C \frac{V_{c}}{\tau_{b}}\left(f\left(P_{\mathrm{A}}\right)-f\left(P_{\mathrm{V}}\right)\right),
$$

may be used.

This simple model makes it possible to recover, without tuning up any parameter, the order of magnitude of the oxygen transfer rate at rest: using $V_{c}=70 \mathrm{~mL}, \tau_{b}=0.75 \mathrm{~s}, P_{\mathrm{A}}=100 \mathrm{mmHg}, P_{\mathrm{V}}=40 \mathrm{mmHg}, \sigma=$ $1.4 \times 10^{-6} \mathrm{~mol} \mathrm{~L}^{-1} \mathrm{mmHg}^{-1}$ and $C=2.2 \times 10^{-3} \mathrm{~mol} \mathrm{~L}^{-1}$, one obtains

$$
Q=1.8 \times 10^{-4} \mathrm{~mol} \mathrm{~s}^{-1}=243 \mathrm{~mL} \mathrm{\textrm {min } ^ { - 1 } ,}
$$

which is accordance with physiological observations (about $250 \mathrm{~mL} \mathrm{~min}{ }^{-1}$ ) that can be found in the literature $[18,43,45]$.

Diffusion limitation on oxygen transfer. The estimate proposed previously was based on the assumption that balance between partial pressures is achieved during the time that blood spends in the neighborhood of alveoli. This assumption has some robustness: under normal conditions, at rest, the time needed to realize this balance is about the third of the available time $\tau_{b}=0.75 \mathrm{~s}$ (see Fig. 5). Yet, this fact might be invalidated in some situations, for example:

1. if $D_{\mathrm{m}}$ is reduced (e.g. in case of emphysema), the process is slowed down, and the time necessary to achieve balance might be larger than $\tau_{b}$ (see the dashed line in Figure 5, which is obtained by reducing $D_{\mathrm{m}}$ by a factor 4);

2. if $\tau_{b}$ is reduced significantly (e.g. during exercise), it may drop below the time necessary to achieve balance;

3. in the same spirit, if $V_{c}$ is larger, the quantity of oxygen needed to achieve balance is larger, and it increases the duration of the process.

The model has to be extended to account for the fact that balance might not be achieved during the available time $\tau_{b}$. It is done simply by solving equation (5) during $\tau_{b}$, starting from an initial value set at the partial pressure of oxygen in the venous blood, namely $P_{\mathrm{V}}$. If we denote by $P_{\mathrm{A}}^{\star}$ the value of $P$ at time $\tau_{b}$, the corresponding oxygen transfer rate is

$$
Q=\frac{V_{c}}{\tau_{b}}[\sigma P+4 C f(P)]_{P_{\mathrm{V}}}^{P_{\mathrm{A}}^{\star}}:=\frac{V_{c}}{\tau_{b}}\left(\sigma P_{\mathrm{A}}^{\star}-\sigma P_{\mathrm{V}}+4 C\left(f\left(P_{\mathrm{A}}^{\star}\right)-f\left(P_{\mathrm{V}}\right)\right)\right) .
$$

Note that one does not have to decide a priori whether we are in the standard situation considered previously (when there is enough time to achieve balance) or not. Indeed, if the speed of the diffusion process is sufficient to achieve balance during $\tau_{b}$, we shall obtain $P_{\mathrm{A}}^{\star}$ very close to $P_{\mathrm{A}}$, and we are brought back to the simple model.

\subsection{Global model}

The coupling between alveolar and plasma compartments is completed by expressing the partial pressure of oxygen in the alveoli as $c \bar{P}_{\text {atm. }}$, where

$$
\bar{P}_{\text {atm. }}=P_{\text {atm. }}-P_{H_{2} O}=760-47=713 \mathrm{mmHg} \quad(=950 \mathrm{hPa})
$$

is the reduced atmospheric pressure. Indeed, as the alveolar air is saturated in water vapor, the actual partial pressure associated to oxygen at a given concentration $c$ is reduced: this saturated vapor pressure of $47 \mathrm{mmHg}$, see e.g. [4], has to be subtracted to standard atmospheric pressure.

\footnotetext{
${ }^{3}$ Indeed, the concentration of oxygen potentially captured by hemoglobin is about $4 C \simeq 9 \mathrm{mmol} \mathrm{L}{ }^{-1}$, whereas the concentration in the plasma which balances alveolar partial pressure is $\sigma P_{\mathrm{A}} \simeq 0.15 \mathrm{mmol} \mathrm{L}^{-1}$ (about 60 times smaller).
} 
The global model for oxygen transfer finally reads

$$
\left\{\begin{aligned}
R \dot{V}+\varphi(V) & =-P \\
\dot{c} & =\frac{1}{V}\left(\dot{V}\left(c^{0}-c\right) H(\dot{V})-Q\right) \\
Q & =\frac{V_{c}}{\tau_{b}}\left(\sigma P_{\mathrm{A}}^{\star}-\sigma P_{\mathrm{V}}+4 C\left(f\left(P_{\mathrm{A}}^{\star}\right)-f\left(P_{\mathrm{V}}\right)\right)\right) \\
P_{\mathrm{A}}^{\star} & =F\left(D_{\mathrm{m}}, V_{c}, P_{\mathrm{V}}, \tau_{b}, P_{\mathrm{A}}\right)
\end{aligned}\right.
$$

where function $F$, which computes the oxygen concentration in plasma at the end of the exchange phase, maps onto the solution at $\tau_{b}$ of the following ordinary differential equation:

$$
\left\{\begin{array}{l}
\dot{P}(t)=\frac{\kappa}{\left(1+4 C \sigma^{-1} f^{\prime}(P(t))\right)}\left(P_{\mathrm{A}}(t)-P(t)\right), t \in\left(0, \tau_{b}\right), \\
P(0)=P_{\mathrm{V}}
\end{array}\right.
$$

with $\kappa=D_{\mathrm{m}} /\left(\sigma V_{c}\right)$ and $P_{\mathrm{A}}(t)=c(t) \bar{P}_{\text {atm. }}$. Note that, as previously mentioned, under normal conditions (healthy person at rest), the kinetics of diffusion does not play any role, and it simply holds

$$
P_{\mathrm{A}}^{\star}=F\left(D_{\mathrm{m}}, V_{c}, P_{\mathrm{V}}, \tau_{b}, P_{\mathrm{A}}\right) \simeq P_{\mathrm{A}}=c \bar{P}_{\mathrm{atm} .},
$$

but the general form has to be kept if one aims at modeling pathological or extreme situations (drastic reduction of $D_{\mathrm{m}}$, reduction of $\tau_{b}$ during exercise, etc.). The global system involving diffusion limitation contains implicit assumptions on the different time scales. In particular, it is considered that actual oxygen transfer occurs at a time scale much smaller than time scales corresponding to variations of $c$ (concentration of oxygen in the lung). In other words, the reaction phenomenon expressed by function $F$ is considered as instantaneous with respect to the first two equations. In practical computations, for a given regime, the relationship between the alveolar concentration and the partial pressure of oxygen in plasma at the end of the exchange phase, namely $P_{\mathrm{A}} \mapsto P_{\mathrm{A}}^{\star}$, may be tabulated. The previous set of equations can be seen as a controlled system: the control variable is $t \mapsto P_{\text {ext. }}(t)$, which corresponds to the action of the diaphragm on the lung. At rest, $P_{\text {ext. }}(t)<0$ during inspiration and $P_{\text {ext. }}(t)=0$ during expiration, which is passive. Actually, the control variable also includes the action of the abdominal muscles during forced expiration, in which case $P_{\text {ext. }}(t)>0$. The output of the process, which can be considered as a utility function, is the mean oxygen transfer rate, usually denoted by $\dot{V}_{\mathrm{O}_{2}}$, corresponding to the average quantity of oxygen (expressed in volume at standard temperature and pressure) transferred into the bloodstream per minute. As we are mainly interested here in the respiration process at a given regime, we shall consider that $P_{\text {ext. }}$ is $T$-periodic (with $T \simeq 5 \mathrm{~s}$ at rest, $T \simeq 3 \mathrm{~s}$ at exercise), so that

$$
\dot{V}_{O_{2}}=\frac{1}{T} \int_{\tau}^{\tau+T} Q(t) \mathrm{d} t
$$

where we assume that the periodic regime has been attained: $\tau$ is chosen sufficiently large in practical computations. The output function on which we shall focus in the section dedicated to numerical experiments is built as follows: consider $T>0$ a time period, $P_{\text {ext. }}$ a $T$-periodic function, we will investigate the behavior of $\dot{V}_{O_{2}}$ as a function of all the parameters:

$$
\dot{V}_{O_{2}}:=\Lambda\left(\varphi, R, T, P_{\text {ext. }}, D_{\mathrm{m}}, V_{c}, P_{\mathrm{V}}, \tau_{b}, C, P_{\text {atm. }}\right)
$$

which is obtained by considering the periodic solution of System (7).

\section{ONE-DIMENSIONAL MODEL FOR DISTRIBUTED OXYGEN TRANSFER}

To account for non-uniformity of the oxygen distribution in the lung, we introduce here a one-dimensional PDE model based on the longitudinal dimension $x$, which corresponds to the distance from the entrance of the 
respiratory tract. To our knowledge, Paiva and Engel [31] were the first ones to propose a one-dimensional PDE in order to investigate the heterogeneity of the gas distribution during the respiratory process: an equation of convection and diffusion for oxygen concentration is proposed although the model contains strong limitations: there is no source term describing the oxygen uptake (oxygen concentration is prescribed in the end of the acinar pathway) and the mechanical process is not considered per se (a sinusoidal volume is prescribed). The approach was extended in [16] to account for oxygen transfer. A similar approach was proposed in [38], including an intensive study of acinar gas mixing and gas exchange from both qualitative and quantitative points of view. See also [41] for a convective diffusive one-dimensional PDE to model the mixing of a marker gas in the lung, and [25] for the description of spirometric maneuvers for a model integrating lung mechanics, pulmonary blood flow, and gas exchange, by means of fitting procedures. Yet the equations which we obtain here present important differences: the (negative) source term describing the gas exchange along the path length in the acinus is estimated according to the nonlinear model presented in the previous section, and the set of equations itself is derived in a different way, so that the obtained equation is fully conservative (unlike the models proposed in $[16,38]$ ), thus providing consistent properties from a physical point of view: maximum principle and Jensen's inequality.

\subsection{The PDE model}

The core of the approach relies on four geometric quantities which can be defined at every level along the one-dimensional representation of the tree (see $[31,39,43,45])$ :

1. Mean length of each generation. The path length, from mouth to the end of the terminal generation, is built upon these data.

2. Cross section, denoted by S. It corresponds to the total cross section available for longitudinal flux. Near the entrance, before the first bifurcation, it is simply the cross section of the trachea, whereas at generation $n$ (after $n$ bifurcation), it is set at $2^{n}$ times the cross section of a single branch. This variable is well-documented in the literature.

3. Lineic volume $\tilde{V}$. It corresponds to the volume of the lung per unit longitudinal length, including the volume of all alveoli connected to the branches at the considered level. Note that for the conductive part, it is equal to the previous variable $\mathrm{S}$, whereas it may be much larger as soon as one enters the acinar zone in which branches are connected to alveoli, beyond the 16 th or 17 th generation.

4. Lineic exchange surface $\tilde{\Sigma}$. It corresponds to the area of the surface of the alveoli available for gas exchange per unit length. It is homogeneous to a length. It is 0 for the conductive part, and starts to increase as soon as one enters the respiratory zone. The total alveolar surface, which is about $130 \mathrm{~m}^{2}$, is distributed over the generations in proportion to the bronchial surface, according to $[17,45]$.

Figure 6 illustrates the one-dimensional representation on the lung, based on the previous geometric considerations. If one denotes by $L$ the "length" of the lung, i.e. the distance along the tree between the entrance of the trachea and the last generation, $x$ runs over the interval $[0, L]$. The quantities defined above are such that

$$
\int_{0}^{L} \tilde{V}(x, t) \mathrm{d} x=V(t)
$$

where $V(t)$ is the total volume of air contained in the lungs, and

$$
\int_{0}^{L} \tilde{\Sigma}(x, t) \mathrm{d} x=\int_{x_{r}}^{L} \tilde{\Sigma}(x, t) \mathrm{d} x=\Sigma(t),
$$

where $x_{r}$ corresponds to the separation between the conductive part and the respiratory part (generation 15 or 16), and $\Sigma$ is the total exchange surface. Denoting by $u$ the longitudinal velocity of the air along the tree, the equations are obtained by expressing the conservation of air volume, considered as incompressible, and local 


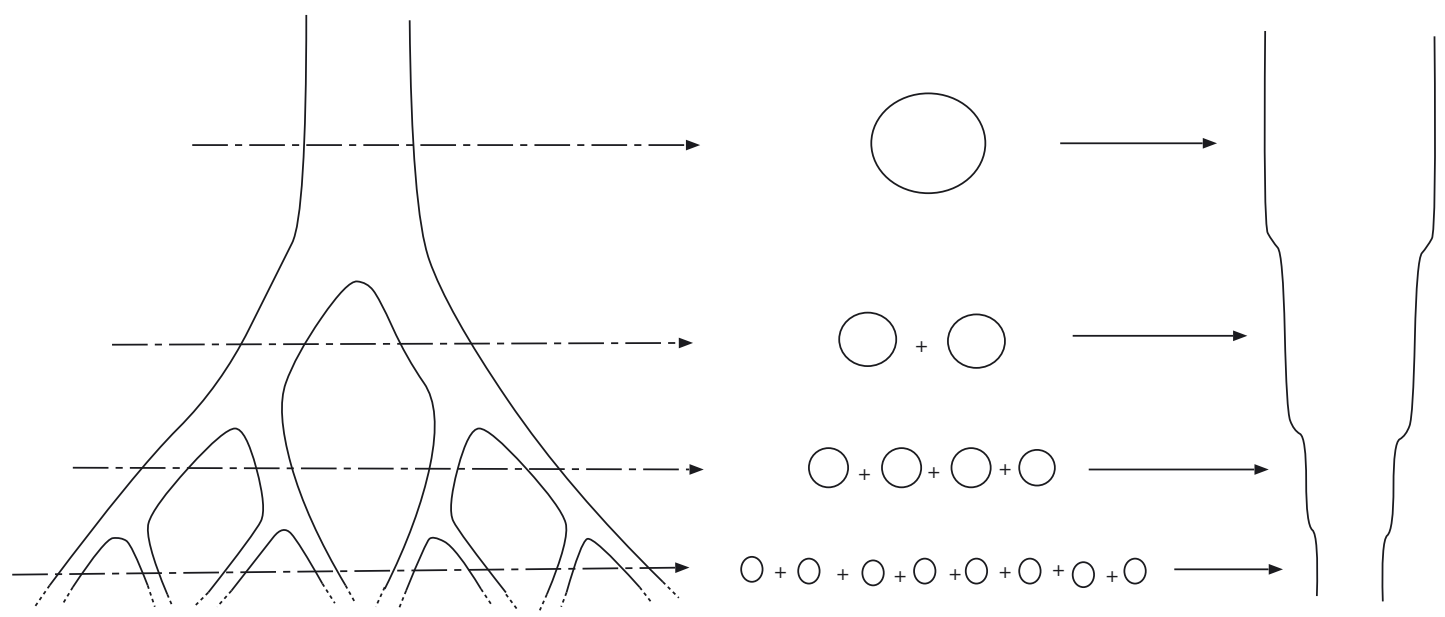

FIgURE 6. Schematic representation and lineic representation of the lung tree.

balance of oxygen:

$$
\begin{aligned}
\partial_{t}(\tilde{V})+\partial_{x}(\mathrm{~S} u) & =0, \\
\partial_{t}(\tilde{V} c)+\partial_{x}(\mathrm{~S} c u)-D_{\mathrm{O}_{2}} \partial_{x}\left(\mathrm{~S} \partial_{x} c\right) & =-\frac{\tilde{\Sigma}}{\Sigma} q(c),
\end{aligned}
$$

where $q(c)$ describes the oxygen transfer into the blood, following the approach developped in the lumped model presented in the previous section (we disregard here diffusion limitation to simplify the presentation, but it could be accounted for, as in the lumped model)

$$
q(c)=\frac{V_{c}}{\tau_{b}}\left(\sigma c \bar{P}_{\mathrm{atm} .}-\sigma P_{\mathrm{V}}+4 C\left(f\left(c \bar{P}_{\mathrm{atm}}\right)-f\left(P_{\mathrm{V}}\right)\right)\right) .
$$

Note the particular form of the transport equation (9). As alveoli can be seen as air reservoirs attached to the ducts, the corresponding volume per unit length is involved in the first term of the transport equation, but transport in the longitudinal direction is based on the sole duct section (lineic volume of branches), which explains that both $\tilde{V}$ and $\mathrm{S}$ appear in this equation. It is crucial to differentiate those two lineic volumes, as they are very different in the respiratory zone $(\tilde{V}=\mathrm{S}$ in the conductive part and $\tilde{V} \gg \mathrm{S}$ near the end of the tree). In the context which we consider here, this transport equation is not actually solved in a standard manner, but it is used to build the instantaneous longitudinal velocity field $u$. Indeed, as detailed in the next section, $\mathrm{S}$ will be considered as constant in time and depending on the space variable only, although we could account within this framework to variations of the duct cross sections. The variations of the total volume are computed according to the lumped mechanical model presented in Section 1, and this volume is then distributed along the acinar zone according to the data given in [43]. The transport equation is then used to recover the field $u$ which ensures volume conservation and oxygen concentration can be determined by solving equation (10).

Remark 3.1. A key assumption under this approach is that the concentration of oxygen is considered uniform in the transverse direction, i.e. it only depends on the distance from the root of the tree. This assumption is actually twofold. As already mentioned, it restricts this approach to the regular situation, where geometric characteristics together with dynamics variables depend on this longitudinal dimension only. It also relies on the fact that, at the level of a single branch, the concentration is uniform in each section, up to the alveoli which might be attached to the branch at the considered level. This latter assumption can be justified if one considers the diffusion coefficient of oxygen in the air, which is $D_{\mathrm{O}_{2}}=2 \times 10^{-5} \mathrm{~m}^{2} \mathrm{~s}^{-1}$. Considering a transverse direction 
of the order $h=1 \mathrm{~mm}$, it gives a characteristic time of $h^{2} / D_{\mathrm{O}_{2}}=0.05 \mathrm{~s}$, so that passive diffusion ensures quasi instantaneous local uniformity in the transverse direction.

Balance of oxygen and link with the lumped model. Global balance of oxygen can be obtained straightforwardly by integrating the second equation of equations (9) and (10) over $[0, L]$ :

$$
\frac{\mathrm{d}}{\mathrm{d} t}\left(\int_{0}^{L}(\tilde{V} c)(x, t) \mathrm{d} x\right)=\mathcal{F}_{\text {in }}(t)-\mathcal{F}_{\text {out }}(t)-Q(t),
$$

where $\mathcal{F}_{\text {in }}(t)$ and $\mathcal{F}_{\text {out }}(t)$ represent the total flux $\mathcal{F}(\cdot, t)$ (with both advective and diffusive contributions) at the entrance $x=0$ and at the end of the tree $x=L$ and the oxygen transfer rate is defined by

$$
Q(t):=\int_{0}^{L} \frac{\tilde{\Sigma}(x, t)}{\Sigma(t)} q(c(x, t)) \mathrm{d} x .
$$

Let us first remark that the diffusive part of the flux at 0 is likely to be much smaller than its advective counterpart as the concentration is fairly uniform in this zone. As for the advective term Suc| $\left.\right|_{x=0}$, note that $\mathrm{S} u=\dot{V}$, so that this term can be related to the considerations we presented at the beginning of Section 2 : we actually considered an inner domain at uniform concentration $c$, an outside world at concentration $c^{0}$, and we followed an upwind principle to account for this source term. A first difference with the lumped model can be pointed out here: as the actual concentration is not constant, and as it is likely to be always maximal at 0 , the lumped model underestimates the advective losses of oxygen during expiration.

Remark 3.2 (Maximum principle). The oxygen transfer rate (last term in Eq. (12)) can be interpreted as the expectancy of $q(c)$ according to the probability measure $\tilde{\Sigma}(x, t) \mathrm{d} x / \Sigma(t)$ on $[0, L]$. Note that, as $q$ is increasing, we have

$$
Q \in\left[q\left(c_{\min }\right), q\left(c_{\max }\right)\right] .
$$

Remark 3.3 (Jensen's inequality). As $c \mapsto q(c)$ is concave in the zone of interest, Jensen's inequality gives

$$
Q(t) \leq q(\bar{c}(t)), \text { with } \bar{c}(t)=\int_{0}^{L} \frac{\tilde{\Sigma}(x, t)}{\Sigma(t)} c(x, t) \mathrm{d} x,
$$

$\bar{c}$ being the instantaneous mean value of the concentration of oxygen. Note also that this inequality is strict as soon as the concentration is not constant, and this latter remark sheds a light on another difference with the lumped model: the latter is based on the fact that the oxygen transfer rate can be estimated as $q(\bar{c})$, where $\bar{c}$ is the mean value of $c$ overall the lung. This approach would be exact if $q$ was affine but, as it is strictly concave in the zone of interest, this expression (on which the lumped model is based) systematically overestimates this transfer.

\subsection{Time and space discretization scheme}

In practice, computations are performed as follows. Consider the lung volume at rest $V_{\mathrm{FRC}}$. Then, the geometric data of a 24-generation lung lead to the definition of piecewise constant functions $\alpha_{V}:(0, L) \rightarrow \mathbb{R}$ defined by

$$
\alpha_{V}(x)=\frac{V^{g}(x)}{L^{g}(x) V_{\mathrm{FRC}}},
$$

where $V^{g}(x)$ and $L^{g}(x)$ respectively denote the volume and path length of generation $g$ such that $x$ is localized in generation $g$. Note that $\alpha_{V}$ satisfies, by construction,

$$
\int_{0}^{L} \alpha_{V}(x) \mathrm{d} x=1
$$


Then,

$$
\tilde{V}(x, t):=\alpha_{V}(x) V(t),
$$

where $V(t)$ denotes the lung volume at time $t$. Thus, $\tilde{V}$ defines the lineic volume of the lung. A similar procedure is used to define the lineic exchange surface $\tilde{\Sigma}$ based on the knowledge of the exchange surface $\Sigma$.

We consider a finite volume discretization. Assume that $\left(c_{i}\right)_{i=1 \ldots N}^{n}$ is the discretized concentration on the cells at time $t_{n}$. At first stage, we solve the mechanic problem which provides $V^{n+1}$ and $\dot{V}^{n+1}$, namely the volume and the variation of volume of the lung at time $t^{n+1}$. We define the lineic variation of volume $\left(\partial_{t} \tilde{V}\right)_{i}^{n+1}$ which is the lineic version of $\dot{V}^{n+1}$. We also define $\tilde{V}_{i}^{n+1}$ and $S_{i}^{n+1}$ as the lineic versions of the lung volume $V^{n+1}\left(=V_{a}^{n+1}+V_{b}^{n+1}\right)$ and bronchial volume $V_{b}^{n+1}$ respectively. Then, we determine the air velocity $u_{i}^{n+1}$ in the airway with an upwinded discretization of the transport equation:

$$
\left(\frac{(\mathrm{S} u)_{i}^{n+1}-(\mathrm{S} u)_{i-1}^{n+1}}{\Delta x}\right) \mathbf{1}_{\left[\dot{V}^{n+1}>0\right]}+\left(\frac{(\mathrm{S} u)_{i+1}^{n+1}-(\mathrm{S} u)_{i}^{n+1}}{\Delta x}\right) \mathbf{1}_{\left[\dot{V}^{n+1}<0\right]}=-\left(\partial_{t} \tilde{V}\right)_{i}^{n+1},
$$

so that the average flow in cells can be determined by supplementing the previous set of equations with the boundary condition $(\mathrm{S} u)_{1}^{n+1}=\dot{V}^{n+1}$ at inspiration and $(\mathrm{S} u)_{N}^{n+1}=0$ at expiration. At second stage, we write the discretized equation on oxygen conservation under the form:

$$
(\tilde{V} c)_{i}^{n+1}+\frac{\Delta t}{\Delta x}\left(\Phi_{i+\frac{1}{2}}-\Phi_{i-\frac{1}{2}}\right)=(\tilde{V} c)_{i}^{n}-\Delta t \frac{\tilde{\Sigma}}{\Sigma} q\left(c^{n}\right),
$$

where $\Phi_{i \pm \frac{1}{2}}$ is an implicit approximation of the total flux (composed of an advective contribution and a diffusive one). Here, the advective flux is upwinded (as in the computation of the air velocity) and the numerical flux is thus defined as

$$
\Phi_{i+\frac{1}{2}}:=(\mathrm{S} u)_{i}^{n+1} c_{i}^{n+1} \mathbf{1}_{\left[\dot{V}^{n+1}>0\right]}+(\mathrm{S} u)_{i+1}^{n+1} c_{i+1}^{n+1} \mathbf{1}_{\left[\dot{V}^{n+1}<0\right]}-D_{O_{2}} \mathrm{~S}_{i+\frac{1}{2}}^{n+1} \frac{c_{i+1}^{n+1}-c_{i}^{n+1}}{\Delta x} .
$$

The boundary conditions depend on the regime: at inspiration, the value of the concentration is prescribed $\left(c_{1}^{n+1}=c^{0}\right.$, where $c^{0}$ is the concentration of fresh air) while, at expiration, a free flux condition is imposed; in both cases, at the end of the lung tree, the flux $\Phi_{N+\frac{1}{2}}$ is zero, meaning that the end of the tree behaves like an insulation boundary (note that the air velocity is already taken to 0 ).

Although classical, this three-point scheme preserves important properties. In particular, constant states (for the concentration) are preserved, due to the fact that the advective flux exactly matches the flux of the airflow in that particular case. As a consequence, the scheme preserves the maximum principle. This is an important feature that may be put in default if the two schemes do not coincide, because the variations of section $\mathrm{S}$ are large. Note that the source term is treated in an explicit way.

\section{Numerical EXPERIMENTs, SEnSitivity ANALysis}

\subsection{Parameters}

We shall consider the following set of assumptions and parameters corresponding to the standard situation of an healthy person at rest, see Table 1 .

During exercise, some parameters have to be modified. In particular, because of the increase of the heart beat, the arterial time period $\tau_{b}$ is set to $0.25 \mathrm{~s}$; blood partial pressure in oxygen is $30 \mathrm{mmHg}$; the mean oxygen transfer rate reaches $2.5 \mathrm{~L} \mathrm{~min}^{-1}$. Capillary volume is multiplied by a factor 3 , and so is the membrane diffusing capacity. Moreover, the external pressure $P_{\text {ext. }}$, accounting for the effort of the diaphragm (and possibly muscles of the abdomen during forced expiration), is $T$-periodic, with

$$
P_{\text {ext. }}(t)=\left\{\begin{array}{l}
P_{\text {insp. }}<0, \text { if } t \in[0, \eta T[ \\
P_{\text {exp. }} \geq 0, \text { if } t \in[\eta T, T[
\end{array}\right.
$$


TABLE 1. Parameters of the models: at rest.

\begin{tabular}{|c|c|c|c|c|}
\hline Parameters & & & & Ref. \\
\hline Membrane diffusing capacity & $D_{\mathrm{m}}$ & 21 & $\mathrm{~mL} \min ^{-1} \mathrm{mmHg}^{-1}$ & {$[4,18]$} \\
\hline Capillary volume & $V_{c}$ & 70 & $\mathrm{~mL}$ & {$[4,18,47]$} \\
\hline Elastance & $E_{0}$ & 3.5 & $\mathrm{cmH}_{2} \mathrm{OL}^{-1}$ & {$[3,4,18]$} \\
\hline Resistance & $R_{0}$ & 2.0 & $\mathrm{cmH}_{2} \mathrm{OsL}^{-1}$ & {$[4,18]$} \\
\hline Arterial time period & $\tau_{b}$ & 0.75 & $\mathrm{~s}$ & {$[4,22,47]$} \\
\hline Reduced atmospheric pressure & $\bar{P}_{\text {atm. }}$. & 713 & $\mathrm{mmHg}$ & {$[4,18]$} \\
\hline Partial pressure of oxygen in the venous blood & $P_{\mathrm{V}}$ & 40 & $\mathrm{mmHg}$ & {$[18,47]$} \\
\hline Total lung capacity & $V_{\mathrm{TLC}}$ & 6.00 & $\mathrm{~L}$ & {$[18]$} \\
\hline Residual volume & $V_{\mathrm{RV}}$ & 1.20 & $\mathrm{~L}$ & {$[18,24]$} \\
\hline sidual capacity & $V_{\text {FRC }}$ & 3.00 & $\mathrm{~L}$ & {$[18,24,39]$} \\
\hline Concentration & $\mathrm{C}$ & $2.2 \times 10^{-3}$ & $\mathrm{~mol} \mathrm{~L}^{-1}$ & {$[4,19]$} \\
\hline Solubility of oxygen in plasma & $\sigma$ & $1.4 \times 10^{-6}$ & $\mathrm{~mol}^{-1} \mathrm{~L}^{-1} \mathrm{mmHg}^{-1}$ & {$[4,22]$} \\
\hline Observablequantities & & & & Ref. \\
\hline Tidal volume & $V_{\mathrm{T}}$ & 0.50 & $\mathrm{~L}$ & {$[39,47]$} \\
\hline Flux & $\dot{V}$ & \pm 0.50 & $\mathrm{Ls}^{-1}$ & {$[47]$} \\
\hline Alveolar partial pressure of oxygen & $P_{\mathrm{A}}$ & 100 & $\mathrm{mmHg}$ & {$[4,18,24,47$} \\
\hline Mean oxygen transfer rate & $\dot{V}_{\mathrm{O}_{2}}$ & 0.25 & $\mathrm{~L} \min ^{-1}$ & {$[18,45]$} \\
\hline
\end{tabular}

At rest, we use $T=5 \mathrm{~s}, \eta=0.35, P_{\text {insp. }}=-2 \mathrm{cmH}_{2} \mathrm{O}$ and $P_{\text {exp. }}=0 \mathrm{cmH}_{2} \mathrm{O}$ since expiration is passive; during exercise, we use $T=3 \mathrm{~s}, \eta=0.50, P_{\text {insp. }}=-5 \mathrm{cmH}_{2} \mathrm{O}$ and $P_{\text {exp. }}=+3 \mathrm{cmH}_{2} \mathrm{O}$.

\subsection{Simulation results for the lumped model}

Numerical results have been performed with this set of parameters in the lumped model. At rest or exercise, observable data such as volume variation, flow, mean alveolar concentration and mean oxygen transfer are reproduced qualitatively and quantitatively. In particular, the model captures the correct magnitude of the mean oxygen transfer rate: $250 \mathrm{~mL} \mathrm{~min}{ }^{-1}$ at rest, and $2.5 \mathrm{~L} \mathrm{~min}^{-1}$ at exercise, in accordance with the values found in the literature, see e.g. [43], p. 284.

In this section we are interested in quantifying the dependence of the utility function $\Lambda$ (see Eq. (8)) upon its parameters. Considering $\Lambda$ as a function of parameters $\left\{\alpha_{i}\right\}_{i}$, we define the sensitivity with respect to $\alpha_{i}$ as

$$
S_{\alpha_{i}}:=\frac{\partial \Lambda}{\partial \alpha_{i}} \frac{\alpha_{i}}{\Lambda}
$$

This dimensionless value quantifies the relative variation of $\Lambda$ with respect to the relative variations of $\alpha_{i}$. Table 2 presents the sensitivity of the oxygen transfer rate, in the framework of our model, with respect to some relevant parameters. Computations have been performed with a standard finite difference approximation.

The most striking feature of these results is the zero sensibility to $D_{\mathrm{m}}$, under normal conditions. It is a direct consequence of the fact, already mentioned, that partial pressures balance in a time which is much (about three times) shorter than $\tau_{b}$. The system is highly robust from this standpoint, as $D_{\mathrm{m}}$ can be reduced threefold without any significant effect on oxygen transfer. Note though that this robustness is typical of resting conditions. When at exercise, with a higher heart frequency ( $\tau_{b}$ close to $0.25 \mathrm{~s}$ ), this security zone does no longer exist, and a decrease of membrane diffusion capacity shall have an immediate effect on oxygen transfer, as suggested in the first line of Table 2.

Another source of degeneracy lies in the flatness of Hills's curve in the neighborhood of the concentration which balances alveolar partial pressure. Disregarding here diffusion limitation and considering that $P_{\mathrm{A}}^{\star}=P_{\mathrm{A}}$, 

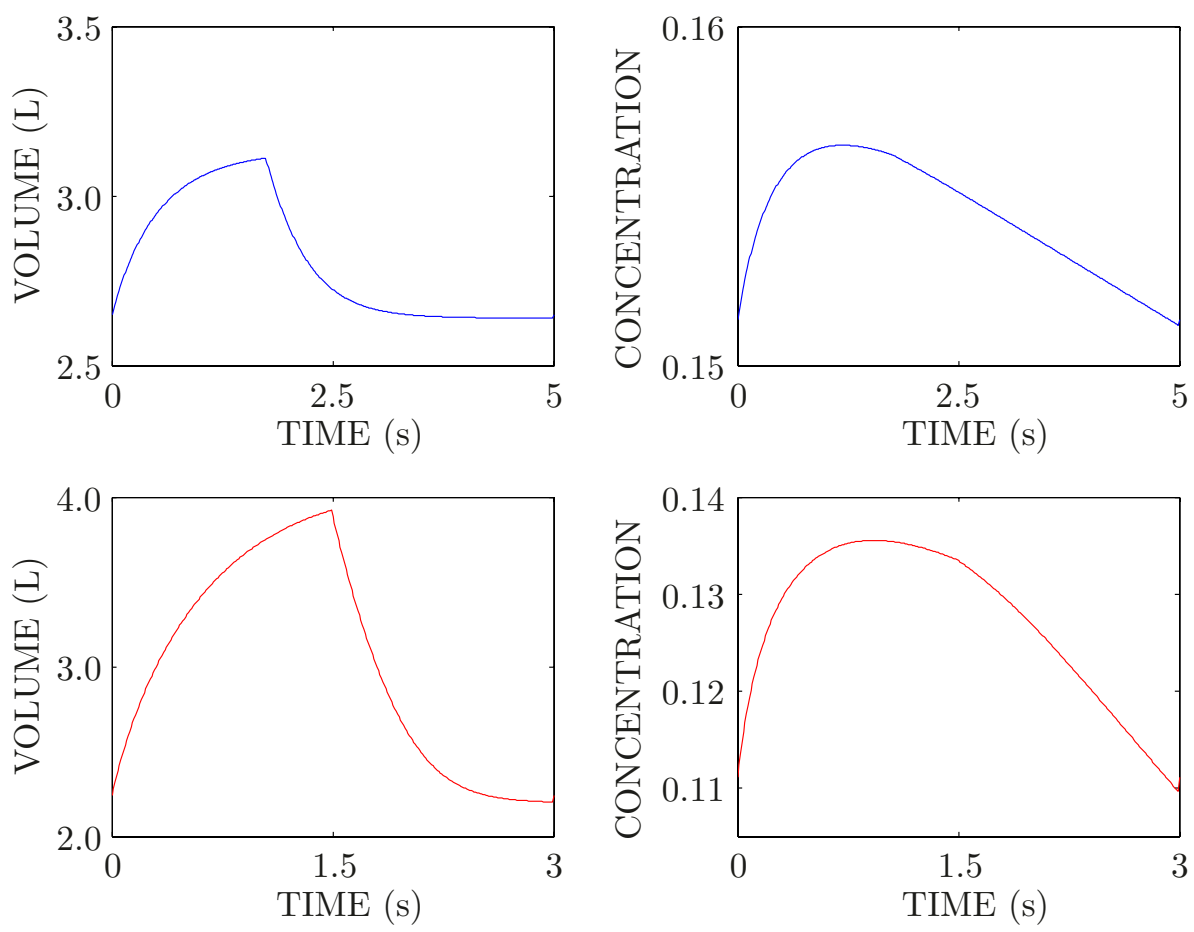

Figure 7. Lumped model: volume and oxygen concentration at rest (top) and during exercise (bottom).

TABLE 2. Sensitivity of the oxygen transfer rate with respect to the parameters of the model.

\begin{tabular}{llrl}
\hline Parameter & & atrest atexercise \\
\hline Membrane diffusing capacity & $D_{\mathrm{m}}$ & 0.00 & +0.35 \\
Elastance & $E$ & -0.07 & -0.26 \\
Resistance & $R$ at $2 \mathrm{cmH}_{2} \mathrm{O} \mathrm{L}^{-1}$ & -0.01 & -0.07 \\
Resistance & $R$ at $4 \mathrm{cmH}_{2} \mathrm{O} \mathrm{L} \mathrm{L}^{-1}$ & -0.04 & -0.28 \\
Resistance & $R$ at $8 \mathrm{cmH}_{2} \mathrm{O} \mathrm{L}^{-1}$ & -0.56 & -3.18 \\
Arterial time period & $\tau_{b}$ & -0.93 & -0.44 \\
Capillary volume & $V_{c}$ & +0.92 & +0.65 \\
\hline
\end{tabular}

the transfer rate

$$
Q=\frac{V_{c}}{\tau_{b}}[\sigma P+4 C f(P)]_{P_{\mathrm{V}}}^{P_{\mathrm{A}}^{\star}}:=\frac{V_{c}}{\tau_{b}}\left(\sigma P_{\mathrm{A}}^{\star}-\sigma P_{\mathrm{V}}+4 C\left(f\left(P_{\mathrm{A}}^{\star}\right)-f\left(P_{\mathrm{V}}\right)\right)\right)
$$

depends on the saturation function $f$ evaluated at $P_{\mathrm{A}}=c \bar{P}_{\text {atm. }}$. As illustrated by Figure 4 , this value is almost constant when oxygen alveolar partial pressure is larger than, say, $70 \mathrm{mmHg}$, which corresponds to an alveolar concentration of $70 / 713 \simeq 10 \%$. The oxygen transfer rate is quite insensitive to the mean alveolar concentration of oxygen as far as it remains above $10 \%$. This explains why the sensitivity to mechanical parameters in the neighborhood of the standard situation is weak. For example, increasing the elastance will mechanically reduce the tidal volume, thus the renewing of oxygen by ventilation, and it will lead to a decrease in the alveolar concentration. But as far as this value (which is about $15 \%$ in standard conditions) remains above $10 \%$, it will 

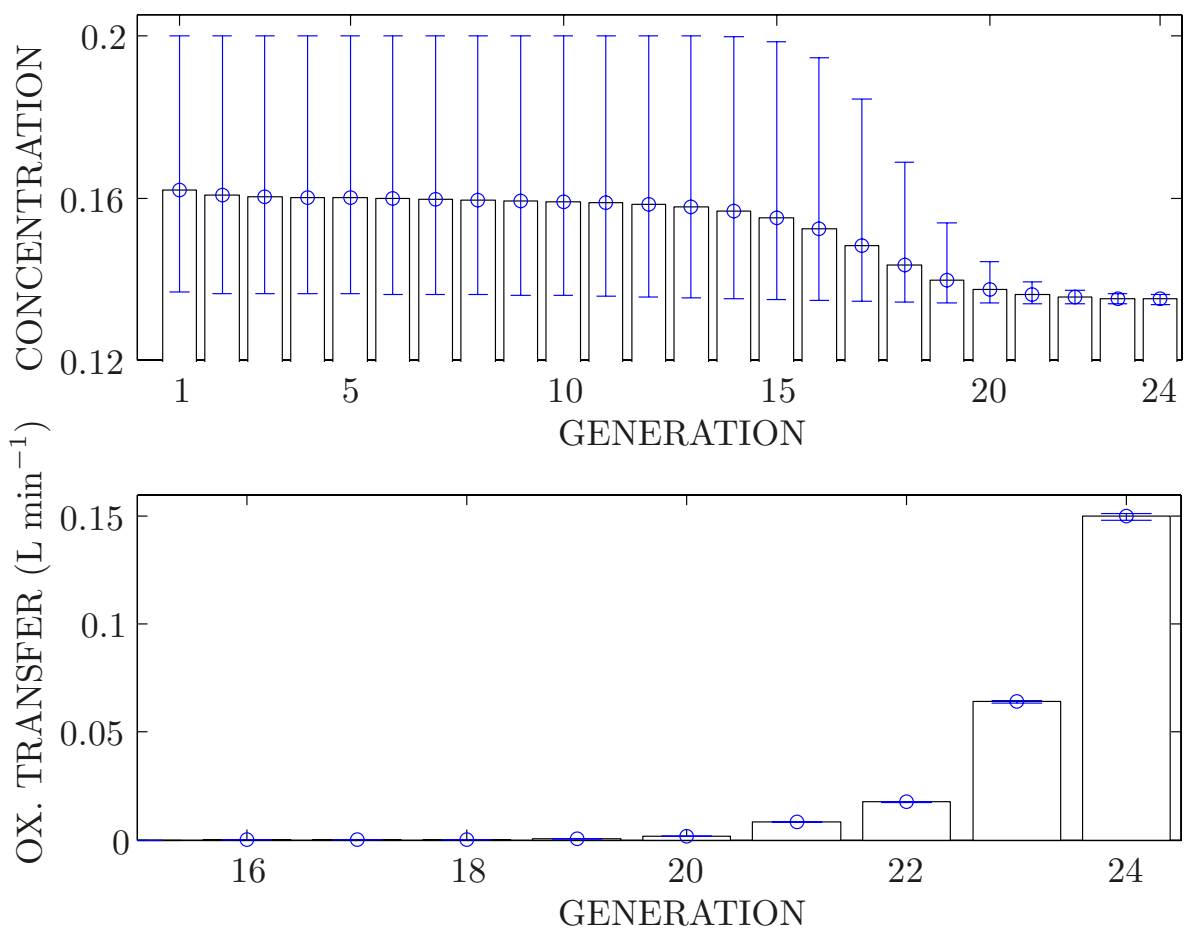

FiguRE 8. One-dimensional model: average concentration and oxygen transfer rate per generation at rest. Circles indicate the mean value over a period, whereas upper (resp. lower) bars indicate the maximal (resp. minimal) value of the concentration during the period.

not affect the oxygen transfer rate in a significant manner. Similarly, increasing slightly the resistance will not affect the oxygen transfer rate. Yet, if this increase of resistance is large enough to really deteriorate the renewal of alveolar oxygen, and reduces it to values smaller than $10 \%$, the sensitivity becomes more important, as it appears in Table 2. For example, the sensitivity with respect to resistance at $R=8 \mathrm{cmH}_{2} \mathrm{O} \mathrm{L}^{-1}$ (about 4 times its standard value) is already 0.5 , whereas it is close to 0 at $2 \mathrm{cmH}_{2} \mathrm{O} \mathrm{L}^{-1}$. The importance of this threshold value around 70 or $75 \mathrm{mmHg}$ for the arterial partial pressure of oxygen, below which a strong deterioration in oxygen transfer is observed, is illustrated in [6]: noninvasive ventilation is considered as successful when it increases the arterial oxygen partial pressure from around $65 \mathrm{mmHg}$ to $80 \mathrm{mmHg}$.

\subsection{Simulation results for the one-dimensional model}

We present here some numerical experiments pertaining to the one-dimensional model. In particular we quantify the heterogeneity of oxygen distribution over the respiratory tract, we investigate what is the contribution to the different acinar generations in the oxygen transfer rate, and we finally illustrate how this model allows to quantify the relative importance of advection and diffusion processes, depending on the generations and the regime which is considered (rest or exercise).

Oxygen heterogeneity. The one-dimensional model leads to values for oxygen transfer rate which are essentially the same as the simpler lumped model: $250 \mathrm{~mL} \mathrm{~min}{ }^{-1}$ at rest and up to $2.5 \mathrm{~L} \mathrm{~min}{ }^{-1}$ at exercise. Additionally, it provides valuable information on the manner oxygen is distributed along the tract. Figure 8 (top) represents, for each generation, the local mean concentration of oxygen, together with extremal values during the ventilation cycle. The mean concentration is quite uniform in the conducting part of the tree up to generation 15, then drops down to a smaller value as it enters the respiratory zone, and remains quasi uniform 
and constant in time in the last generations. Note that the value of $14 \%$ that is obtained in this zone, where most part of transfer takes place, corresponds to a partial pressure of $0.14 \times 713 \mathrm{mmHg} \simeq 100 \mathrm{mmHg}$, in accordance with the available literature, see $[4,18,24,47]$. Figure 8 (bottom) details the different contributions to oxygen transfer rate of the different generations. In particular, the interest of the one-dimensional model is to be able to capture the well-documented value of the average oxygen concentration in alveoli, which is about $14 \%$, whereas the lumped model tends to overestimate this average value. As expected, the most part of the exchange area lies in the last two generations so that the transfer is essentially concentrated near the very end of the tree and oxygen transfer outside peripheral acinar regions is mostly negligible. From a qualitative point of view, our results are close to the ones described in [38] in the sense that diffusion screening $[12,14,34]$ does not significantly impair acinar gas exchange function. In particular, oxygen transfer does not play a significant role in the decrease in partial pressure of oxygen along the path length. But unlike previous models, our criteria selection depends not only on the heterogeneity of the oxygen concentration $c$ along the path length, but also on a quantitative study of the oxygen transfer that may include nonlinear effects such as diffusion limitation. The same experiment has been led under exercise, see Figure 9: the variations of oxygen concentration are larger than under resting conditions; in terms of oxygen transfer, there is no significant difference with the rest regime, as the oxygen flux is nearly constant in each generation.

Figures 10 and 11 show the temporal variation in oxygen concentration at the trachea, acinus entrance, and acinus periphery over two steady-state breaths. They appear to be in good agreement with the results presented in [38]: the sharp transitions at the trachea at end inspiration and end expiration are due to the square-wave breathing function $t \mapsto P(t)$. At the acinus entrance, the oxygen concentration increases after a delay following the start of an inspiration as fresh air reaches the acinus through the deadspace. The oxygen concentration in the acinus periphery only varies a little.

Transition between advection and diffusion. In [39] Sznitman investigates, by means of local estimates of the Péclet number, the relative importance of convection and diffusion in the transport of oxygen in the pulmonary acinus. In order to focus on the relative importance of advective and diffusive contributions in the gas transfer process along the path length, we propose the following approach. At any location $x \in[0, L]$, the instantaneous flux of oxygen through $x$ can be decomposed onto two contributions

$$
\Phi(x, t)=\underbrace{(\mathrm{S} u c)(x, t)}_{\text {advective }}-\underbrace{\left(D_{\mathrm{O}_{2}} \mathrm{~S} \partial_{x} c\right)(x, t)}_{\text {diffusive }}=\Phi_{a}(x, t)+\Phi_{d}(x, t) .
$$

The mean flux over the period $T$

$$
\bar{\Phi}(x)=\frac{1}{T} \int_{0}^{T} \Phi(x, t) \mathrm{d} t=\frac{1}{T} \int_{0}^{T}(\mathrm{~S} u c)(x, t) \mathrm{d} t-\frac{1}{T} \int_{0}^{T}\left(D_{O_{2}} \mathrm{~S} \partial_{x} c\right)(x, t) \mathrm{d} t=\bar{\Phi}_{a}(x)+\bar{\Phi}_{d}(x)
$$

corresponds to the quantity of oxygen which has been transferred to the blood in the zone which corresponds to interval $[x, L]$. It is then natural to define

$$
\theta_{a}(x)=\frac{\bar{\Phi}_{a}(x)}{\bar{\Phi}_{a}(x)+\bar{\Phi}_{d}(x)}
$$

as an indicator of the importance of advection at $x$, whereas $\theta_{d}=\bar{\Phi}_{d} /\left(\bar{\Phi}_{a}+\bar{\Phi}_{d}\right)=1-\theta_{a}$ pertains to diffusion. In this way, $\theta_{a}+\theta_{d} \equiv 1$ and we expect that $\theta_{a}$ is close to 1 in the upper part of the bronchial tree (i.e. in the non-alveolated area) whereas $\theta_{d}$ becomes predominant in the end of the bronchial tree (in particular, in the last generation), with a transition that occurs in the acinus. Figure 12 highlights this behavior: the transition between advection and diffusion is localized at generation 18 at rest, and generation 21 at exercise: muscular efforts leading to an increase of the flow during exercise have a direct impact over the advective flux. 

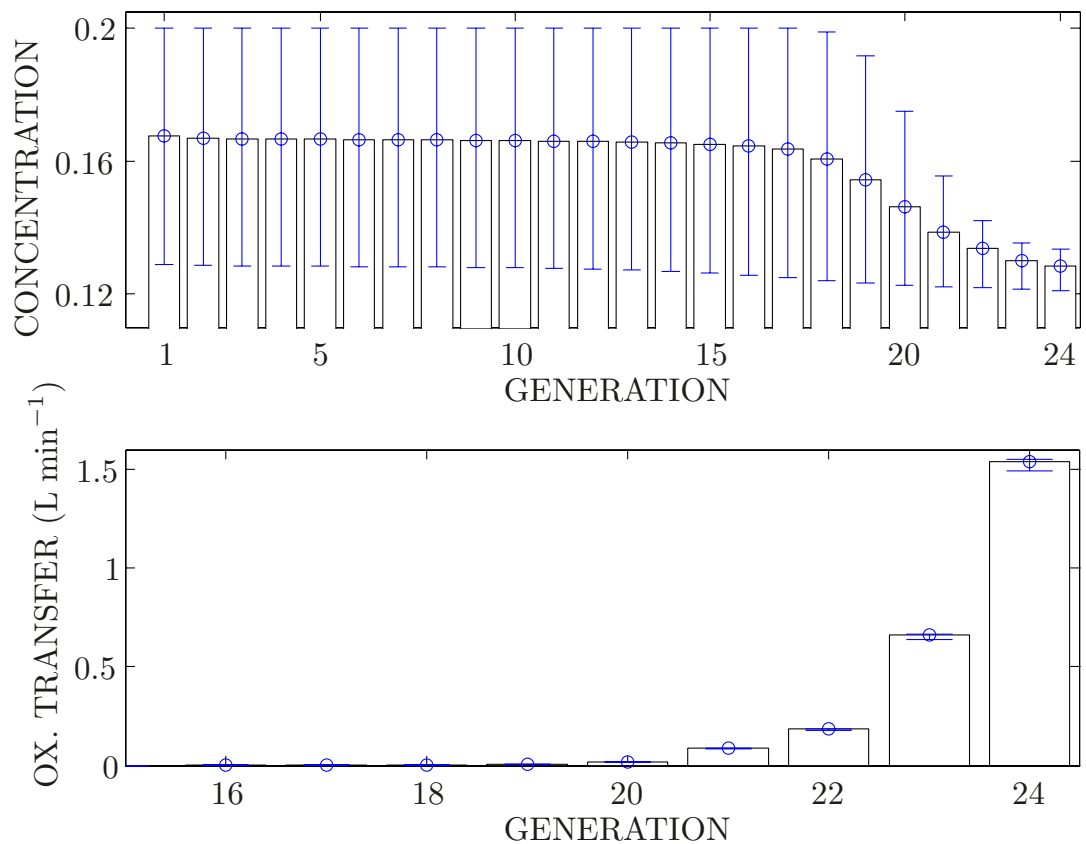

FiguRE 9. One-dimensional model: average concentration and oxygen transfer rate per generation during exercise. Circles indicate the mean value over a period, whereas upper (resp. lower) bars indicate the maximal (resp. minimal) value of the concentration during the period.

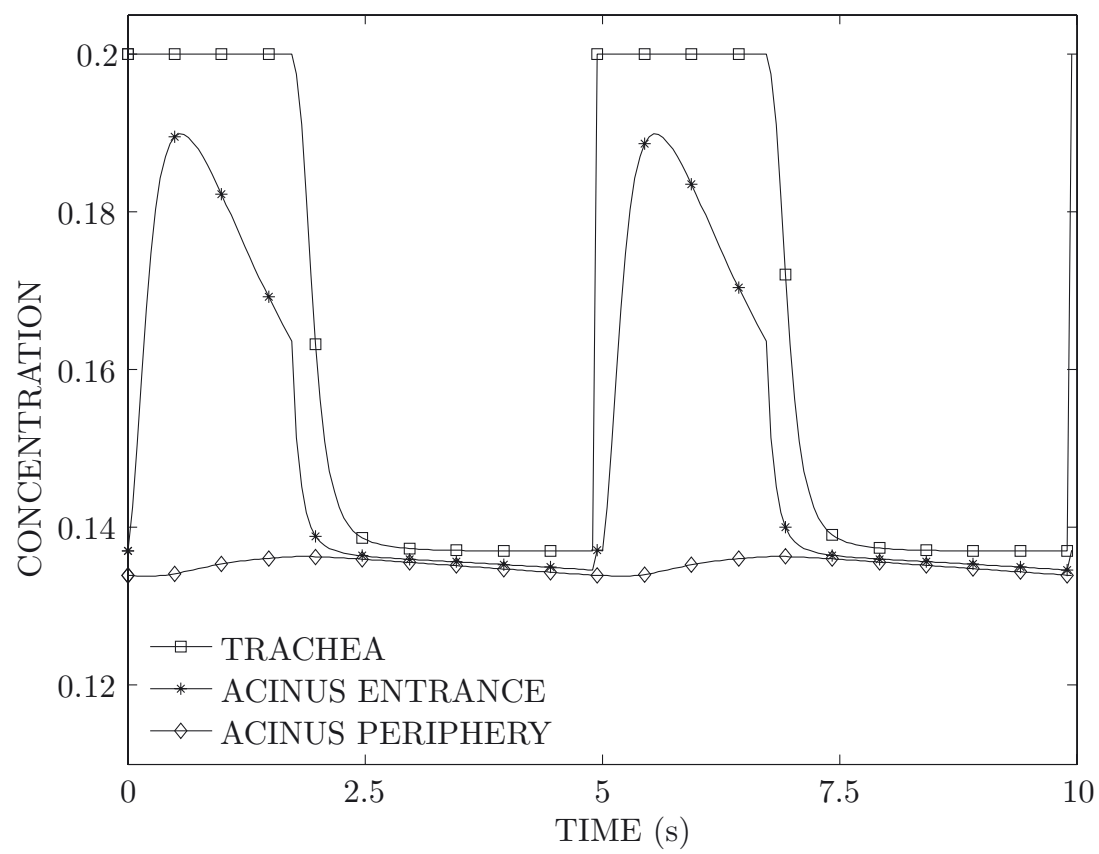

FigURE 10. At rest: temporal variation of oxygen concentration over 2 breaths, at the trachea, acinus entrance, acinus periphery. 


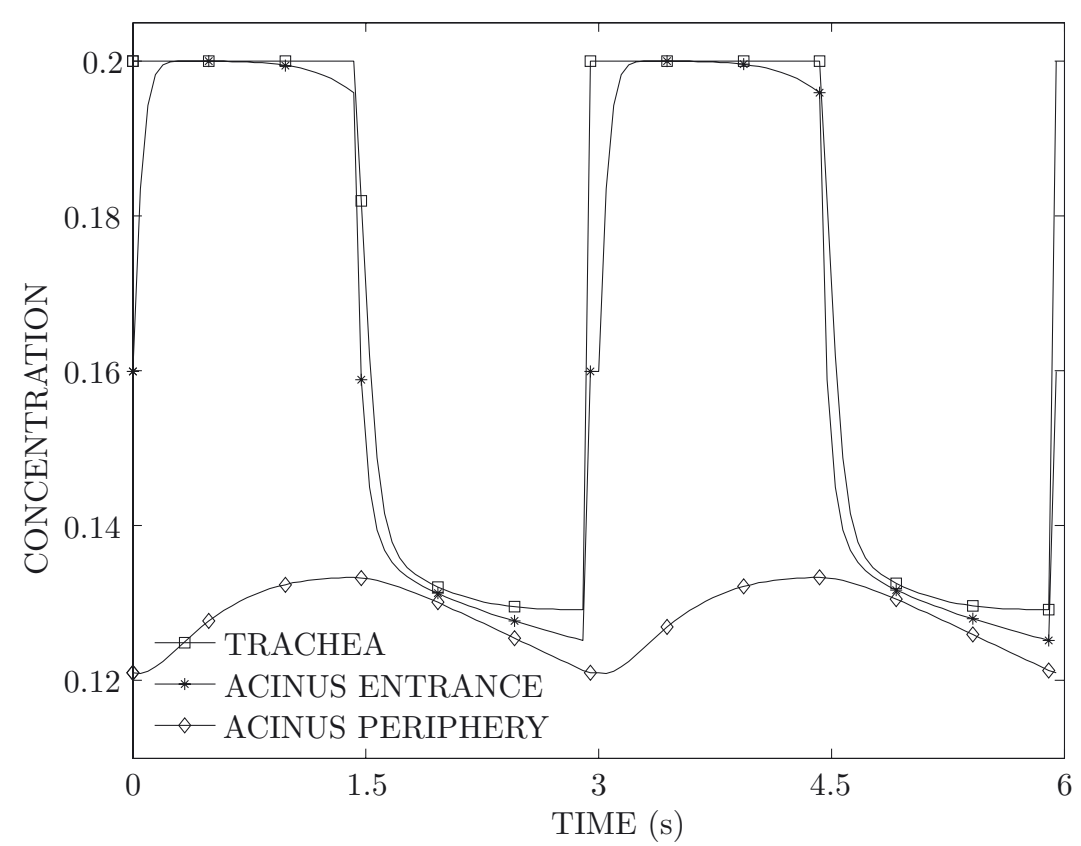

FiguRE 11. At exercise: temporal variation of oxygen concentration over 2 breaths at the trachea, acinus entrance, acinus periphery.
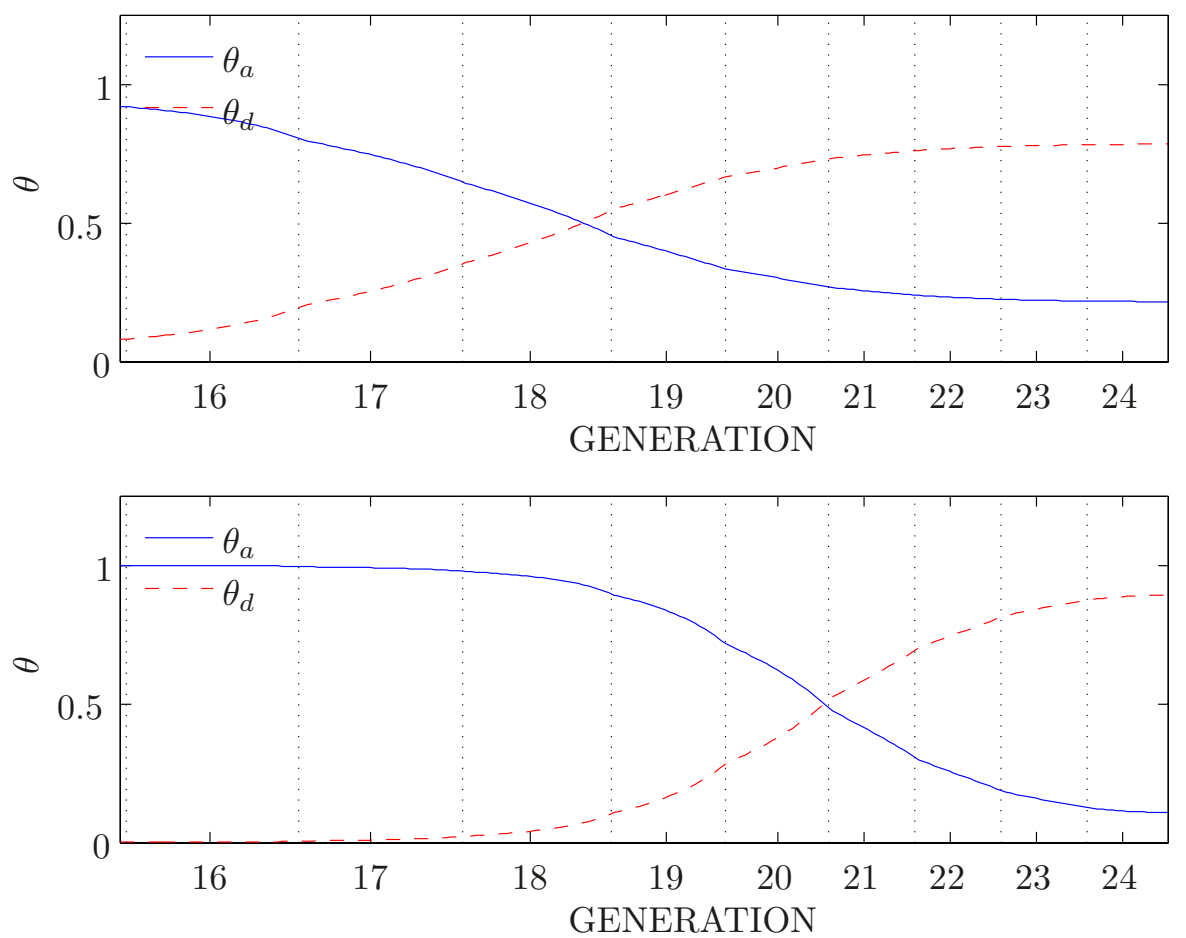

FIgURE 12. Transition between advection and diffusion in the acinus at rest (top) and during exercise (bottom). 


\section{Discussion}

\subsection{Main results}

Diffusion limitation in extreme maneuvers. The diffusing capacity of the alveolar-capillary membrane does not affect the oxygen transfer rate in the normal regime but, as it decreases (e.g. because of emphysema) below a critical value, it becomes a significant parameter: this phenomenon explicitly results from the diffusion limitation. In this way, at rest or exercise, the oxygen transfer is optimal in a standard situation as blood that goes out of the capillaries is fully satured in oxygen; but the oxygen transfer is much more sensitive to parameters such as elastance or membrane diffusing capacity in a situation at exercise than at rest, because of diffusion limitation combined with a set of physiological data which are closer to critical values at exercise than at rest.

Acinar heterogeneity. A one-dimensional model has been derived, based on mass-flow conservative properties in order to investigate the acinar heterogeneity in terms of oxygen concentration and oxygen transfer into the blood. It has led us to investigate the diffusional screening phenomenon, i.e. the possibility that oxygen transfer might be significantly affected by the fact that the peripheral exchange area participates poorly to oxygen transfer at rest, thereby providing a natural reserve of transfer capacity for exercise condition. We do not recover this effect in the regime we consider. In particular we show that, at rest, although the oxygen concentration is slightly smaller in terminal alveoli, transfer mainly occurs in the acinar periphery, at rest and at exercise.

\subsection{Limitations of the model}

Mechanical model. The mechanical model we considered is quite simple. We assumed in particular that the resistance of the respiratory system is constant. In fact, bronchi have elastic walls and the lumen of the bronchi are not constant during the ventilation, see e.g. $[15,30]$. It is possible, in our model, to model nonlinear effects of the resistance. Indeed, it is known that the resistance varies with the volume (it is roughly proportional to the inverse of the volume): elementary calculus based on the Poiseuille law (see e.g. [29,47]) together with experimental studies (see e.g. [24], page 85 ) lead to consider that the conductance $1 / R$ depends linearly on the volume. It is then natural to assume

$$
R:=R(V)=\frac{V_{\mathrm{FRC}}}{V} R_{0},
$$

where $V_{\mathrm{FRC}}$ is the volume at static equilibrium (functional residual volume), and $R_{0}$ the corresponding resistance. This dependence might be very significant in extreme situations (e.g. spirometry), yet it does not affect much the mechanical behavior in the situation we considered. Besides, in terms of oxygen transfer, the significant variable is the tidal volume (it conditions the rate of renewal), and this tidal volume can be checked to be almost insensitive to the resistance, under normal conditions.

Lumped transfer model. The oxygen transfer model relies on quantities which are well documented in the literature: capillary volume $V_{c}$, transit time $\tau_{b}$, membrane diffusion capacity $D_{\mathrm{m}}$, and oxygen partial pressure in the venous blood $P_{\mathrm{V}}$. We did not investigate the effect of capillary closing on oxygen transfer onto the blood, and how it may affect the sense given to the capillary volume $V_{c}$, which was assumed here to be constant. The transit time $\tau_{b}$ plays a critical role in the model, it carries the information pertaining to the way blood circulates in capillaries, in the neighborhood of alveoli. Weibel [43] estimates that, at rest, an erythrocyte spends less than one second on the alveolar capillary, and the reference value of 0.75 at which we set $\tau_{b}$ is widely documented in the literature. During strenuous exercise, this transit time is considered to be reduced to the third of its value at rest (a value of 0.3 is given in Weibel [43] during strenuous exercise). Like most authors, we considered here that a decrease of the heart beat period (during exercise) induces a proportional decrease of $\tau_{b}$. Note those quantities are considered as intrinsically different in [9], but our model, in its present form, does not allow such 
a distinction. All the parameters of the transfer model are supposed to be known and constant, for a given regime (rest or exercise).

Transverse heterogeneity. A first source of heterogeneity lies in the fact that the actual lung is not perfectly symmetric. It is known in particular that when a branch separates onto two subbranches, the dimensions of the daughter branches are unbalanced, see [27]. Another source of non-uniformity in the transverse direction is related to inertial effects. It was pointed out in [28] that, because of inertia, the Poiseuille velocity profile is deformed in the first generations, which might induce heterogeneity in fluxes, depending on bifurcation angles. Note though that this effect is restricted to the first generations, where inertial effect are most significant. Beyond these deterministic sources of dissymmetry, geometrical data have some intrinsic inter- and intrasubject variability. In particular, for terminal bronchioles (between generation 12 and 15), lengths and diameters variations go up to $60 \%$, as reported in [37]. For all those reasons, advective transfer of air in the respiratory tract is likely to deviate from the perfect symmetry that we assumed to build the one-dimensional model. Note that the incidence of neglecting this transverse heterogeneity upon oxygen transfer can be estimated. Firstly, the one-dimensional model is likely to over-estimate the oxygen transfer rate (because of the concavity of the saturation function, see Rem. 3.3), compared to a more sophisticated model which would account for transverse heterogeneity. Secondly, according to Remark 3.3, the oxygen transfer rate is interpreted as the expectancy (or mean value) of $q(c)$ according to some probability measure. This principle is quite general: a more sophisticated model, accounting for transverse heterogeneity, would lead to a similar property, with a different measure. As a consequence, whenever concentration $c$ ranges in a zone where $q$ is almost flat, the oxygen transfer rate is quite insensitive to heterogeneities in oxygen concentrations. The latter remark concerns normal conditions (with an oxygen alveolar partial pressure larger than $90 \mathrm{mmHg}$, so that the saturation function is almost flat). In critical situations, for example for breathing in altitude, smaller values of alveolar oxygen partial pressure could be obtained, attaining zones where the saturation function is stiffer. In the latter situation, accounting for transverse heterogeneities would have significant effects on the computed oxygen transfer. Note also that, if the assumption of transverse homogeneity is ruled out, the transition between advection and diffusion could not be properly defined for the global tree (as it is illustrated by Fig. 12), but may depend upon the subtree in which it is estimated.

Localized impairing. Let us add that the one-dimensional model does not allow, in its present form, to account for a localized impairing of the alveolar-capillary membrane, or to large heterogeneities in terms of perfusion. As an example, panacinar emphysema is likely to affect the acini belonging to the lower lobes of the lung, and an accurate description of this type of situation necessitates a more sophisticated transfer model, possibly built as a combination of interconnected one-dimensional models, coupled to a full mechanical model to account for heterogeneities in the local compliance of the lung tissues.

Ventilation-perfusion ratio. Other types of heterogeneities have not been taken into account in this article:

- regional differences in ventilation: it is well-known [47] that the lower regions of the lung are better ventilated than the upper regions, because of the effects of gravity on the lung;

- regional differences in perfusion: blood flow is highly variable within the human lung, due to the difference between the arterial pressure and the alveolar pressure, and may be affected by change of posture and exercise.

Thus, the different perfusion zones and the differences in ventilation have not been considered, although alteration (increase or decrease) of the ventilation-perfusion ratio may have a strong impact on the overall gas exchange efficiency. It is well-known [47] that the ventilation-perfusion ratio decreases down the lung. The integration of such heterogeneities shall be considered as major improvements of our model. 


\section{CONCLUSion}

We presented an integrated model for oxygen transfer onto the blood, which relies solely on quantities with a clear physiological meaning. The lumped version of the model allows to recover well-documented orders of magnitude for oxygen transfer rate, in different regimes, and reproduces possible diffusion limitation when both extreme regimes and membrane impairing are considered. The one-dimensional version of the model allows to investigate the effects of longitudinal heterogeneity of oxygen concentration upon the mean transfer rate. Oxygen concentration is found to be smaller in the last generations of the acini, without affecting significantly oxygen transfer: most part of it is realized in the periphery, in all the regimes that we considered.

Acknowledgements. The authors would like to thank Anne Devys and Christophe Roussel for fruitful discussions.

\section{REFERENCES}

[1] E. Agostoni and R.E. Hyatt, Static behavior of the respiratory system, in Handbook of physiology, edited by S.R. Geiger, 2nd edition. American Physiological Society, Bethesda (1986) 113-130.

[2] D.V. Bates, C.J. Varvis, R.E. Donevan and R.V. Christie, Variations in the pulmonary capillary blood volume and membrane diffusion component in health and disease. J. Clin. Invest. 39 (1960) 1401-1412.

[3] R. Begin, A.D. Renzetti Jr., A.H. Bigler and S. Watanabe, Flow and age dependence of airway closure and dynamic compliance. J. Appl. Physiol. 38 (1975) 199-207.

[4] A. Ben-Tal, Simplified models for gas exchange in the human lungs. J. Theor. Biol. 238 (2006) 474-495.

[5] C. Brighenti, G. Gnudi and G. Avanzolini, A simulation model of the oxygen alveolo-capillary exchange in normal and pathological conditions. Physiol. Meas. 24 (2003) 261-275.

[6] L. Brochard, J. Mancebo, M. Wysocki, F. Lofaso, G. Conti, A. Rauss, G. Simonneau, S. Benito, A. Gasparetto, F. Lemaire, D. Isabey and A. Harf, Noninvasive ventilation for acute exacerbations of chronic obstructive pulmonary disease. N. Engl. J. Med. 333 (1995) 817-822.

[7] J.E. Cotes, D.J. Chinn and M.R. Miller, Lung function: Physiology, Measurement and Application in Medicine, 6th edition. Blackwell Publishing Ltd. (2006).

[8] J.E. Cotes, D.J. Chinn, Ph. Quanjer, J. Roca and J.C. Yernault, Standardization of the measurement of transfer factor (diffusing capacity). Eur. Respir. J. suppl 16 (1993) 41-52.

[9] Crandall, E.D. and R.W. Flumerfelt, Effect of time-varying blood flow on oxygen uptake in the pulmonary capillaries. Appl. Physiol. 23 (1967) 944-953.

[10] The lung: Scientific Foundations, edited by R.G. Crystal, J.B. West, E.R. Weibel and P.J. Barnes, 2nd edition. LippincottRaven Press, Philadelphia 2 (1997).

[11] W.A. Eaton, E.R. Henry, J. Hofrichter and A. Mozzarelli, Is cooperative oxygen binding by hemoglobin really understood?. Nat. Struct. Biol. 6 (1999) 351-358.

[12] M. Felici, M. Filoche and B. Sapoval, Diffusional screening in the human pulmonary acinus. J. Appl. Physiol. 94 (2003) 2010-2016.

[13] M. Felici, M. Filoche and B. Sapoval, Renormalized random walk study of oxygen absorption in the human lung. Phys. Rev. Lett. 92 (2004) 068101.

[14] M. Felici, M. Filoche, C. Straus, T. Similowski and B. Sapoval, Diffusional screening in real 3D human acini - a theoretical study. Respir. Physiol. Neurobiol. 145 (2005) 279-293.

[15] M. Filoche and M. Florens, The stationary flow in a heterogeneous compliant vessel network. J. Phys. Conf. Ser. 319 (2011) 012008.

[16] A. Foucquier, Dynamique du transport et du transfert de l'oxygène au sein de l'acinus pulmonaire humain. Ph.D. thesis, École Polytechnique (2010).

[17] P. Gehr, M. Bachofen and E.R. Weibel, The normal human lung: ultrastructure and morphometric estimation of diffusion capacity. Respir. Physiol. 32 (1978) 121-140.

[18] A.C. Guyton and J.E. Hall, Textbook of medical physiology, 9th edition. W.B. Saunders Co, Philadelphia (1996).

[19] M.P. Hlastala and A.J. Berger, Physiology of Respiration, 2nd edition. Oxford University Press, Oxford (2001).

[20] C. Hou, S. Gheorghiu, M.-O. Coppens, V.H. Huxley and P. Pfeifer, Gas diffusion through the fractal landscape of the lung: How deep does oxygen enter the alveolar system? in Fractals in Biology and Medicine, edited by G.A. Losa, D. Merlini, T.F. Nonnenmacher, E.R. Weibel. Basel: Birkhäuser IV (2005) 17-30.

[21] J.M.B. Hughes, Pulmonary gas exchange. in Lung Function Testing, edited by R. Gosselink and H. Stam. European Respiratory Monograph 10 (2005) 106-126.

[22] J. Keener and J. Sneyd, Mathematical Physiology. Interdisciplinary Applied Mathematics. Springer (1998).

[23] G.R. Kelman, Digital computer subroutine for the conversion of oxygen tension into saturation. J. Appl. Physiol. 21 (1966) 1375-1376.

[24] J.D. Kibble and C. Halsey, Medical Physiology, The Big Picture. McGraw Hill (2009). 
[25] C.H. Liu, S.C. Niranjan, J.W. Clark, K.Y. San, J.B. Zwischenberger and A. Bidani, Airway mechanics, gas exchange, and blood flow in a nonlinear model of the normal human lung. J. Appl Physiol. 84 (1998) 1447-1469.

[26] S. Martin, T. Similowski, C. Straus and B. Maury, Impact of respiratory mechanics model parameter on gas exchange efficiency. ESAIM Proc. 23 (2008) 30-47.

[27] B. Mauroy and P. Bokov, Influence of variability on the optimal shape of a dichotomous airway tree branching asymmetrically. Phys. Biol. 7 (2010) 016007.

[28] B. Mauroy, M. Filoche, J.S. Andrade Jr. and B. Sapoval, Interplay between flow distribution and geometry in an airway tree. Phys. Rev. Lett. 90 (2003) 14.

[29] B. Mauroy, M. Filoche, E.R. Weibel, and B. Sapoval, An optimal bronchial tree may be dangerous. Nature 427 (2004) $633-636$.

[30] B. Mauroy and N. Meunier, Optimal Poiseuille flow in a finite elastic dyadic tree. ESAIM: M2AN 42 (2008) 507-534.

[31] M. Paiva and L.A. Engel, Model analysis of gas distribution within human lung acinus. J. Appl. Physiol. 56 (1984) $418-425$.

[32] J. Piiper and P. Scheid, Respiration: alveolar gas exchange. Annu. Rev. Physiol. 33 (1971) 131-154.

[33] F.J. Roughton and R.E. Forster, Relative importance of diffusion and chemical reaction rates in determining rate of exchange of gases in the human lung, with special reference to true diffusing capacity of pulmonary membrane and volume of blood in the lung capillaries. J. Appl. Physiol. 11 (1957) 290-302.

[34] B. Sapoval and M. Filoche, Role of diffusion screening in pulmonary diseases. Adv. Exp. Med. Biol. 605 (2008) $173-178$.

[35] B. Sapoval, M. Filoche and E.R. Weibel, Smaller is better - but not too small: a physical scale for the design of the mammalian pulmonary acinus. Proc. Natl. Acad. Sci. USA 99 (2002) 10411.

[36] T. Similowski and J.H.T. Bates, Two-compartment modelling of respiratory system mechanics at low frequencies: gas redistribution or tissue rheology? Eur. Respir. J. 4 (1991) 353-358.

[37] T.T. Soong, P. Nicolaides, C.P. Yu and S.C. Soong, A statistical description of the human tracheobronchial tree geometry. Respir. Physiol. 37 (1979) 161-72.

[38] A.J. Swan and M.H. Tawhai, Evidence for minimal oxygen heterogeneity in the healthy human pulmonary acinus. J. Appl. Physiol. 110 (2011) 528-537.

[39] J. Sznitman, Convective gas transport in the pulmonary acinus: comparing roles of convective and diffusive lengths. J. Biomech. 42 (2009) 789-792.

[40] C. Tantucci, A. Duguet, P. Giampiccolo, T. Similowski, M. Zelter and J.-P. Derenne, The best peak expiratory flow is flowlimited and effort-independent in normal subjects. Am. J. Respir. Crit. Care Med. 165 (2002) 1304-1308.

[41] M.H Tawhai and P.J. Hunter, Characterising respiratory airway gas mixing using a lumped parameter model of the pulmonary acinus. Respir. Physiol. 127 (2001) 241-248.

[42] E.R. Weibel, Morphometry of the human lung, Springer Verlag and Academic Press, Berlin, New York (1963).

[43] E.R. Weibel, The pathway for oxygen, Harvard University Press (1984).

[44] E.R. Weibel, Design and morphometry of the pulmonary gas exchanger, in The lung: scientific foundations, 2nd edition, edited by R.G. Crystal, J.B. West, E.R. Weibel, P.J. Barnes. Lippincott-Raven Press, Philadelphia 1 (1997) $1147-1157$.

[45] E.R. Weibel, B. Sapoval and M. Filoche, Design of peripheral airways for efficient gas exchange. Resp. Phys. Neur. 148 (2005) $3-21$.

[46] E.R. Weibel, How does lung structure affect gas exchange? Chest 83 (1983) 657-665.

[47] J.B. West, Respiratory physiology: the essentials, Baltimore: Williams and Wilkins (1974).

[48] J.P. Whiteley, D.J. Gavaghan and C.E. Hahn, Some factors affecting oxygen uptake by red blood cells in the pulmonary capillaries. Math. Biosci. 169 (2001) 153-172. 